\title{
REPRESENTATIONS OF FINITE GROUPS OF LIE TYPE
}

\author{
BY CHARLES W. CURTIS ${ }^{1}$
}

The representation theory of a group $G$ over the field of complex numbers involves two problems: first, the construction of the irreducible representations of $G$; and second, the problem of expressing each suitably restricted complex valued function on $G$, as a linear combination (or a limit of linear combinations), of the coefficients of the irreducible representations.

For example, if $G$ is the additive group of real numbers mod 1 (the one-dimensional torus), one considers integrable functions on $G$, or what is the same thing, integrable periodic functions of period 1 on the additive group of real numbers. In this case the irreducible representations of $G$ are given by the exponential functions $x \rightarrow e^{2 \pi i k x}$, where $k$ is an integer, and are the continuous homomorphisms from $G$ into the multiplicative group of complex numbers. The expression of an integrable function in terms of the irreducible representations $\left\{e^{2 \pi i k x}\right\}$ is the Fourier expansion of $f$,

$$
f(x) \sim \sum_{-\infty}^{\infty} a_{k} e^{2 \pi i k x},
$$

where the Fourier coefficients $a_{k}$ are given in terms of $f$ and the representations by the formulas

$$
a_{k}=\int_{-\infty}^{\infty} f(t) e^{2 \pi i k t} d t, \quad k=0, \pm 1, \pm 2, \ldots
$$

For such a group, and, more generally, for locally compact groups and Lie groups, the solution of the problems of representation theory involves both the construction of the irreducible representations, and the question of under what circumstances the Fourier expansion converges, and in particular, whether it converges to the function.

The subject of harmonic analysis, to which the preceding considerations lead, is an old and pervasive part of mathematics, pursued for more than a century, and still full of life. It has fostered many triumphs, and certainly one of the greatest has been Harish-Chandra's contributions to harmonic analysis on semisimple Lie groups (see, for example, Warner [12]). ${ }^{2}$

The paper is based on an address delivered at the Annual Meeting of the American Mathematical Society in Atlanta, Georgia, on January 6, 1978; received by the editors January 25, 1979.

AMS (MOS) subject classifications (1970). Primary 20C15; Secondary 20G05, 20 G40.

Key words and phrases. Chevalley group, $(B, N)$-pair, cuspidal characters, Hecke algebras, generic ring, generic degrees, reductive groups over finite fields, MacDonald conjectures, Deligne-Lusztig virtual characters.

${ }^{1}$ Work on this article was partially supported by the NSF Grant MCS76 07015.

${ }^{2}$ References preceded by $I$ are found in the short list at the end of the Introduction; numbered references not preceded by $I$ are at the end of the article. 
The purpose of this paper is to describe some recent progress on the problem of constructing the irreducible representations, in the field of complex numbers, of finite groups of Lie type.

Finite groups of Lie type are the counterparts, in finite group theory, of semisimple Lie groups. They are the groups constructed by Chevalley in his Tôhoku Journal paper of 1955, and twisted versions of them. They include the classical groups over finite fields, and families of finite simple groups arising from the exceptional simple Lie algebras. Their structural properties have been thoroughly analyzed from the points of view of finite group theory, finite geometrical systems, and algebraic groups defined over finite fields.

Their representation theory, as described above, involves mainly the construction of the irreducible representations; the convergence of the Fourier expansion, etc. offers little resistance, as we are dealing with functions on a finite set.

Nevertheless, the problem of constructing their representations and characters $^{3}$ in the field of complex numbers, begun by Frobenius in 1896 [I1, Bd. 3, p. 29] for the groups $P S L_{2}(p)$, is still not completely solved.

New methods have been introduced, however, by Deligne and Lusztig, of such originality and strength, that decisive progress has been made. This article is an attempt to survey the present situation, with some background on various ways of approaching the groups themselves, some earlier results on the representation theory, and an introduction to the contributions of Deligne and Lusztig.

There are several fascinating, and, to the author anyway, still somewhat mysterious, features of the theory. Hopefully these points are discussed in a more satisfactory way later on, but it seems appropriate to mention them here, so the reader will be looking out for them.

The first is the remarkable fact, first shown by Harish-Chandra (see \$2.2), and later given an explicit and detailed form in the conjectures of MacDonald (see §4.3), that a common set of principles govern the behavior of the irreducible (unitary) representations of semisimple Lie groups (which are infinite-dimensional) and finite groups of Lie type (where the groups are discrete and the representations finite-dimensional).

Another noteworthy development begins with the fact that finite groups of Lie type are associated with a finite group generated by reflections, and a finite field. The finite groups generated by reflections (or the finite Coxeter groups) include the symmetric groups, the hyperoctahedral groups (which are the symmetry groups of $n$-dimensional cubes), and five groups associated with the exceptional Lie algebras, which have appeared previously in other disguises (for example as the group associated with 27 lines on a cubic surface). The representations of these groups are all realizable in the rational field, and the characters, and in most cases the representations, have been constructed over a long period of time by Frobenius and Young, in the case of the symmetric and the hyperoctahedral groups, and by Kondo and Frame

\footnotetext{
${ }^{3}$ See $\$ 2.1$ below for an introduction to some of the main facts about representations and characters of finite groups which will be needed for this paper.
} 
for the exceptional ones. ${ }^{4}$ Their work has proved to be of crucial importance for the problem of decomposing induced representations of finite groups of Lie type into their irreducible components, using the important new concept of a generic algebra of a finite Coxeter group, defined by Tits (see \$3.2).

A third theme arises from the fact that the groups are associated with finite fields, so that their representation theory produces information relating finite fields and the field of complex numbers. This subject, as Weil pointed out in his article [82], originated with Gauss' work on the number of solutions of certain congruences, which he related to Gauss sums. Deligne and Lusztig defined actions of the finite groups of Lie type on algebraic varieties defined over finite fields, which in turn define representations on the $l$-adic cohomology of the varieties. The methods they discovered for computing the characters of these representations are closely related to the cohomological methods, whose existence was conjectured by Weil in [82], for counting the rational points on varieties over finite fields (see §4.3).

As a final note, we remark that characters of finite groups have been traditionally applied to investigate the structure of the groups. In the present situation, the character theory again leads to new group theoretical information, which we illustrate in $\$ 3.2$ with an outline of the determination of the doubly transitive permutation representations of the finite groups of Lie type. Another application, on the number of unipotent classes in reductive groups over finite fields, was given by Lusztig [52].

This paper is restricted to a survey, without proofs, of general results, which apply to all finite groups of Lie type. The groups exist in nature, however, as individual families of classical groups, exceptional groups, twisted groups, etc. Often the most interesting results concern the special behavior of individual families of groups, which is invisible in the general situation. For these results, and proofs of the general theorems, we have provided some references, without attempting completeness. A guide to the literature is also included, indicating appropriate references for each section. Of special importance are the articles of Deligne and Lusztig [25], and Lusztig's own survey [56], to which it is hoped the last part of this article will serve as an introduction.

\section{REFERENCES}

[I1] F. G. Frobenius, Gesammelte Abhandlungen, Bd. 1, 2, 3, Springer-Verlag, Berlin, 1968.

[I2] G. Warner, Harmonic analysis on semi-simple Lie groups. I, II, Die Grundlehren der Math. Wissenschaften, Bd. 188, 189, Springer-Verlag, New York, 1970.

[I3] A. Young, Quantitative substitutional analysis. I-VIII, Proc. London Math. Soc. (1) 33 (1901), 97-146; (1) 34 (1902), 361-397; (2) 28 (1928), 255-292; (2) 31 (1930), 253-272; (2) 31 (1930), 273-288; (2) 34 (1932), 196-230; (2) 36 (1933), 304-368; (2) 37 (1934), 441-495.

\footnotetext{
${ }^{4}$ See [11, Bd. 3, p. 148] and [13] for the work of Frobenius and Young, and [46], [30] and [31] for the work of Kondo and Frame.
} 


\section{TABLE OF CONTENTS}

1. Finite groups of Lie type.

1.1 Chevalley groups.

1.2 Finite groups with $(B, N)$-pairs.

1.3 Parabolic subgroups.

2. Representations and characters.

2.1 Basic principles of representation theory.

2.2 The philosophy of cusp forms. The main problems.

3. Decomposition of induced characters.

3.1 Centralizers and Hecke algebras.

3.2 Principal series representations. Generic degrees.

4. Construction of cuspidal characters.

4.1 Reductive groups over finite fields.

4.2 Cuspidal characters of $G L_{n}(q)$ via the Brauer lift.

The Steinberg representation.

4.3 Representations on $l$-adic cohomology.

The solution of the MacDonald conjectures.

4.4 Unipotent characters.

1. Finite groups of Lie type. This section contains an outline of two descriptions of finite groups of Lie type: a brief account of their construction as Chevalley groups, and their axiomatic description as groups with $(B, N)$ pairs. A third characterization, in terms of algebraic groups, will be given in §4. The notations $Z$ for the integers, $Q$ for the rationals, and $\mathbf{C}$ for the complex field will be used.

1.1 Chevalley groups. Let $\mathrm{g}$ be a semisimple Lie algebra over the complex field $\mathbf{C}$, and let $\Phi=\{\alpha, \beta, \ldots\}$ be a root system of $g$ with respect to a Cartan subalgebra $\mathfrak{h}$. For each root $\alpha \in \Phi$, let $\mathrm{g}_{\alpha}$ be the one-dimensional root subspace corresponding to $\alpha$. Chevalley proved that there exists a basis of $\mathfrak{g}$ consisting of elements $X_{\alpha} \in \mathfrak{g}_{\alpha}$, for $\alpha \in \Phi$, together with a basis of $\mathfrak{h}$ such that the structure constants are all rational integers. The set of $Z$-linear combinations of these basis elements forms a Lie algebra $g_{Z}$ over $Z$, which is a $Z$-form of $\mathrm{g}$.

It was then proved by Kostant [47] that from the Chevalley basis of $\mathfrak{g}$, it is possible to construct a $Z$-form $\mathfrak{U}_{Z}$ of the universal enveloping algebra $\mathfrak{U}$ of $\mathfrak{g}$, such that $\mathfrak{U}_{Z}$ is generated, as an algebra over $Z$, by the elements

$$
\left\{X_{\alpha}^{m} / m !, \alpha \in \Phi, m=1,2, \ldots\right\} .
$$

Now let $\pi: \mathfrak{g} \rightarrow \operatorname{End}_{\mathbf{C}}(M)$ be a representation of $\mathfrak{g}$ by linear transformations on a finite-dimensional vector space $M$ over $\mathbf{C}$. Then $\pi$ can be extended to a representation $\pi: \mathfrak{U} \rightarrow \operatorname{End}_{\mathbf{C}}(M)$, so that $M$ becomes a left $\mathfrak{U}$-module. A $Z$-form $M_{Z}$ of $M$ is called admissible if $M_{Z}$ is stable under left multiplication by elements of $\mathfrak{u}_{Z}$; admissible $Z$-forms can be shown always to exist, and on such a $Z$-form $M_{Z}$, we have, for each $\alpha \in \Phi,\left(X_{\alpha}^{m} / m !\right) M_{Z}=0$, for all sufficiently large $m$. Therefore, for an indeterminate $t$ over $Z$, the endomorphism

$$
x_{\alpha}^{Z}(t)=\exp \left(t X_{\alpha}\right)=\sum_{i=0}^{\infty} t^{i}\left(X_{\alpha}^{i} / i !\right)
$$


is defined on $M_{Z}^{Z[t]}$, and its matrix with respect to a $Z$-basis of $M_{Z}$ will have entries which are polynomials in $t$ with coefficients in $Z$.

Now let $k$ be an arbitrary field, and let $\mathrm{g}_{Z}, M, M_{Z}, x_{\alpha}^{Z}(t)$ be as above. Then

$$
\mathfrak{g}_{k}=k \otimes_{Z} \mathfrak{g}_{Z},
$$

becomes a Lie algebra over $k$, and acts on the $k$-vector space

$$
M_{k}=k \otimes_{Z} M_{Z},
$$

so that $M_{k}$ is a $g_{k}$-module. For each root $\alpha$, we may substitute elements of $k$ for the indeterminate $t$ in the matrix of $x_{\alpha}^{Z}(t)$, and obtain endomorphisms

$$
x_{\alpha}(t): M_{k} \rightarrow M_{k}, \quad t \in k,
$$

which are in fact automorphisms of $M_{k}$ (because they are given by an exponential series). Note that the action of $\mathfrak{u}_{Z}$ on $M_{Z}$, is such that it is possible to construct automorphisms of the vector spaces $M_{k}$ using the exponential series without having problems with the denominators $m$ ! in the series, even in case $k$ is a field of characteristic $p>0$.

Definition. Let $\mathrm{g}_{k}, M_{k}, x_{\alpha}(t) \in \operatorname{End}_{k}(M)$ be as above. For each $\alpha \in \Phi$, let $U_{\alpha}=\left\{x_{\alpha}(t): t \in k\right\}$, and let

$$
G_{\pi, k}=\left\langle U_{\alpha}: \alpha \in \Phi\right\rangle .
$$

The group $G_{\pi, k}$ is the Chevalley group associated with the representation $\pi$ of $g$ and the field $k$.

Chevalley constructed the groups $G_{\pi, k}$ for all fields $k$, where $\pi$ is the adjoint representation ad: $\mathfrak{g} \rightarrow \operatorname{End}_{\mathrm{c}}(\mathrm{g})$, given by ad $X(Y)=[X, Y]$, for $X, Y \in \mathrm{g}$. He proved that if $\mathrm{g}$ is a simple Lie algebra, then the Chevalley group $G_{\mathrm{ad}, k}$ is a simple group for all fields $k$ (with some exceptions for the field $k$ of two or three elements and certain Lie algebra of rank one or two). He realized that this construction placed under one roof the classical linear groups, whose structure had been worked out over finite fields by Dickson [26], and by Artin and Dieudonné in general (see [27]), and the families of simple groups corresponding to the exceptional Lie algebras of types $G_{2}, F_{4}, E_{6}, E_{7}$ and $E_{8}$.

Not long after Chevalley's paper had appeared, several authors found that other families of simple groups could be constructed by twisting a Chevalley group $G_{\pi, k}$, that is, forming the subgroup $G_{\pi, k}^{\sigma}$ of elements in $G_{\pi, k}$ left fixed by a suitable automorphism $\sigma$ of the abstract group $G_{\pi, k}$. Among the groups obtained in this way were the unitary groups, certain twisted orthogonal groups, and infinite families of finite simple groups discovered by Suzuki and Ree arising from Chevalley groups of type $B_{2}, G_{2}$, and $F_{4}$, for certain finite fields $k$ of characteristic 2 and 3 .

The groups described above, for finite fields $k$, are the finite groups of Lie type. They occupy a central position in finite group theory because, taken together, they include all known infinite families of finite simple groups, besides the alternating groups.

1.2 Finite groups with $(B, N)$-pairs. The finite Chevalley groups and their twisted versions have a common subgroup structure, which provided the basis of the analysis, by Chevalley and his successors, of the structure of the groups. The subgroup structure was axiomatized by Tits, as follows, and leads 
to our second, axiomatic, description of finite groups of Lie type.

Definitions. A $(B, N)$-pair (or Tits system) in a group $G$ is a pair of subgroups $B$ and $N$ such that $B$ and $N$ generate $G$, and the intersection $T=B \cap N$ is a normal subgroup of $N$ such that the factor group $W=N / T$ is a finite group generated by involutions $\left\{w_{1}, \ldots, w_{n}\right\}$, satisfying the following conditions, for $w \in W$ and $w_{i} \in\left\{w_{1}, \ldots, w_{n}\right\}$ :

$$
w_{i} B w \subset B w B \cup B w_{i} w B
$$

and

$$
w_{i} B w_{i} \neq B \text {. }
$$

(Here we are using the notation $w B$, for $w \in W$, to denote the coset $n B$, where $n \in N$ is an element such that $n T=w$ in $W$; the coset $w B$ is independent of the choice of $n$ because $T \subseteq B$.)

The subgroup $B$ in a $(B, N)$-pair is called a Borel subgroup; the group $W$ is called the Weyl group; the elements $\left\{w_{1}, \ldots, w_{n}\right\}$ are the distinguished generators of $W$, and the number $n$ is called the rank of the $(B, N)$-pair.

All the finite groups of Lie type discussed in $\S 1.1$ have $(B, N)$-pairs. A good example to keep in mind is the $(B, N)$-pair of rank $n-1$ in the Chevalley group $G_{\pi, k}$ where $\pi$ is the identity representation of the Lie algebra $\mathfrak{g}$ consisting of all linear endomorphisms of trace zero on an $n$-dimensional vector space. In this case, $\pi(x)=x$, for $x \in \mathfrak{g}$, and $G_{\pi, k}$ is isomorphic to the group $S L_{n}(k)$ of $n$ by $n$ matrices over $k$ with determinant 1. A $(B, N)$-pair in $G_{\pi, k}$ is given as follows:

$$
\begin{aligned}
& \left.B=\left\{\left[\begin{array}{lll}
* & & * \\
& \ddots & \\
0 & & *
\end{array}\right]\right\} \quad \text { (upper triangular matrices in } S L_{n}(k)\right), \\
& N=\{\text { all monomial matrices of determinant } 1\}
\end{aligned}
$$

(where a monomial matrix has exactly one nonzero entry in each row and column).

$$
\begin{gathered}
T=B \cap N=\{\text { all diagonal matrices of determinant } 1\} . \\
W=N / T \cong S_{n}, \text { the symmetric group of order } n !
\end{gathered}
$$

The distinguished generators $\left\{w_{1}, \ldots, w_{n-1}\right\}$ of $W$ in this case can be taken as the images in $W$ of the matrices

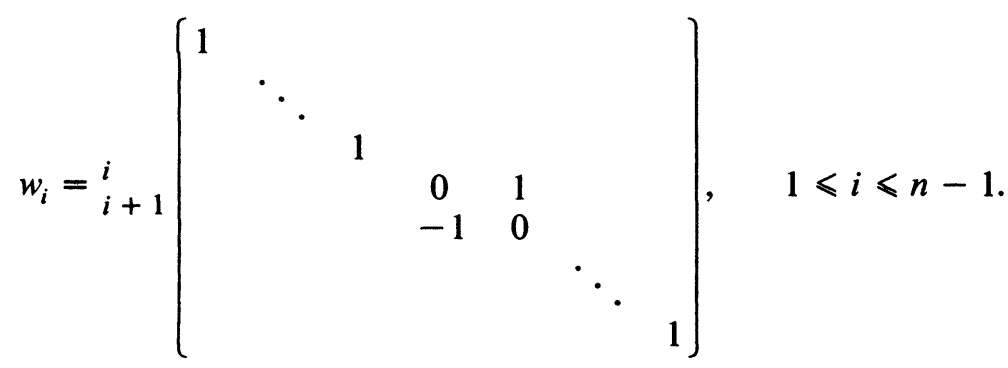

Notice that the same construction yields a $(B, N)$-pair in the general linear group $G L_{n}(k)$. Similar constructions yield $(B, N)$-pairs in the other classical 
groups such as the symplectic groups $S p_{2 k}(k)$ and the orthogonal groups $O_{n}(k)$.

For an arbitrary Chevalley group $G_{\pi, k}(\$ 1.1)$, a $(B, N)$-pair is obtained as follows. Let $\Phi_{+}$denote a positive system of roots in $\Phi$, and let $U=\left\langle U_{\alpha}\right.$ : $\left.\alpha \in \Phi_{+}\right\rangle$. Then for a Borel subgroup $B$, we may take the normalizer $N(U)$ of $U$ in $G_{\pi, k}$. The subgroup $B$ has a semidirect product decomposition $B=T U$, where $T$ is the subgroup of $G_{\pi, k}$ consisting of all elements acting as diagonal transformations with respect to a suitable basis of $M_{k}$. For the subgroup $N$, we may then take the normalizer of $T$ in $G_{\pi, k}$.

Using the axioms, it can be shown that the $(B, B)$-double cosets in a group $G$ with a $(B, N)$-pair are parametrized by the elements of the Weyl group.

(1.2.1) THEOREM (BRUHAT DECOMPOSITION). Let $G$ be a group with $a$ $(B, N)$-pair. Then

$$
G=\bigcup_{w \in W} B w B
$$

with $B w B=B w^{\prime} B$ if and only if $w=w^{\prime}$ in $W$.

The Bruhat decomposition in $G L_{n}(k)$ can be exhibited as follows. Let $g \in G L_{n}(k)$ be arbitrary. Left multiplication by elements of $B$ allow us to perform enough of the elementary row transformations so that the nonzero leading elements in each row of $b g$, for suitable $b \in B$, appear in different positions. It follows that for some permutation matrix $n \in N$, we have $n b g \in B$, and hence $g \in B n^{-1} B$.

A deeper result about $(B, N)$-pairs in finite groups is that the Weyl group is always a Coxeter group, that is, $W$ has a presentation with generators and relations given by

$$
W=\left\langle w_{1}, \ldots, w_{n}:\left(w_{i} w_{j}\right)^{m_{i j}}=1, \text { with } m_{i i}=1 \text { for all } i\right\rangle .
$$

Moreover, the indecomposable Coxeter groups that can occur are precisely those associated with the known families of finite simple groups of Lie type (where a Coxeter group is indecomposable if it is impossible to separate the distinguished generators $\left\{w_{1}, \ldots, w_{n}\right\}$ into two nonempty sets for which the generators in one set commute with the generators of the other).

Using his theory of buildings, Tits was then able to prove that not only the Weyl groups, but the finite groups with $(B, N)$-pairs themselves, are characterized by the axioms. More precisely, he proved that every finite simple group with a $(B, N)$-pair of rank at least 3 is isomorphic to a Chevalley group, described in $\$ 1.1$.

For our purposes, a more detailed axiomatic description will be used, for which a classification has been achieved without restrictions on the rank.

Definition. Let $p$ be a prime. A finite group $G$ has a split $(B, N)$-pair of characteristic $p$, and Weyl group $W$, provided that $G$ has a $(B, N)$-pair with Weyl group $W, B$ is the semidirect product of a normal $p$-subgroup $U$ and the subgroup $T=B \cap N, T$ is an abelian group of order prime to $p$, and $T=\bigcap_{n \in N} n B n^{-1}$.

The groups described in $\S 1.1$ all have split $(B, N)$-pairs as defined above. In $G L_{n}(k)$ (or $S L_{n}(K)$ ) the subgroup $U$ is the subgroup of $B$ consisting of 
upper triangular matrices with 1's on the diagonal. Finite abelian groups $T$ of order prime to $p$ also satisfy the definition; in this case $W=\{1\}, B=N=$ $T$, and $U=\{1\}$.

It is essential for the study of the representations not to consider finite groups of Lie type in isolation from one another, but as members of infinite families.

DEFINITION. A family of finite groups of Lie type consists of the following data. We are given a finite Coxeter group $W$, and an infinite set of prime powers $\{q\}$, called characteristic powers. For each characteristic power $q$, there corresponds a group $G(q)$ in the family, such that $G(q)$ is a finite group with a split $(B, N)$-pair of characteristic $p$ and Weyl group $W$, where $p$ is the prime dividing $q$.

For example, $\left\{G L_{n}(q)\right\}$, for fixed $n$, is a family of finite groups of Lie type associated with the Coxeter group $S_{n}$. In this case (and for all untwisted Chevalley groups) the set of characteristic powers consists of all prime powers. For the families of twisted groups discovered by Suzuki and Ree, however, the characteristic powers associated with the family consist of powers of a single prime (either 2 or 3 ).

1.3 Parabolic subgroups. Let $\{G(q)\}$ be a family of finite groups of Lie type, with the common Weyl group $W$, and let $B(q)$ denote a Borel subgroup of $G(q)$, for each $q$. The Bruhat decomposition (1.2.1)

$$
G(q)=\bigcup B(q) w B(q) \quad(w \in W)
$$

is independent of $q$, in the sense that the double cosets are parametrized by a set independent of $q$ (namely $W$ ).

Some other subgroups with this property are the parabolic subgroups, defined, for a finite group $G$ with a $(B, N)$-pair, as the subgroups $P$ containing the Borel subgroup $B$, and their conjugates. Those containing $B$ will sometimes be called standard parabolic subgroups. For each standard parabolic subgroup $P$ there exists a uniquely determined subset $J$ of the distinguished generators of $W$, such that $P=B W_{J} B$, where $W_{J}$ is the parabolic subgroup of $W$ generated by the elements of $J$.

(1.3.1) Theorem. Let $P=B W_{J} B$ and $P^{\prime}=B W_{J} B$ be standard parabolic subgroups. There exists a bijection

$$
P \backslash G / P^{\prime} \leftrightarrow W_{J} \backslash W / W_{J^{\prime}},
$$

given explicitly by the map

$$
W_{J} w W_{J^{\prime}} \rightarrow B W_{J} w W_{J^{\prime}} B=P w P^{\prime}
$$

Note that the preceding result extends the Bruhat decomposition (1.2.1). A further basic property of the parabolic subgroup is also, in a certain sense, independent of $q$. For a finite group $X$, we recall that $O_{p}(X)$ denotes the unique maximal normal $p$-subgroup of $X$. Each finite group $G(q)$ in a given family of finite groups of Lie type can be shown to satisfy the condition

$$
O_{p}(G(q))=\{1\}
$$

where $p$ is the characteristic of $G(q)$. On the other hand, $O_{p}(B(q))=U(q)$, 
and

$$
B(q)=T(q) U(q) \quad \text { (semidirect product). }
$$

Moreover $B(q)$ is precisely the normalizer in $G(q)$ of its $O_{p}$-subgroup. These properties (of $G(q)$ and $B(q)$ ) carry over to all parabolic subgroups.

(1.3.2) ThEOREM. Let $P=B W_{J} B$ be a standard parabolic subgroup of $G=G(q)$. Let $V=O_{p}(P)$, where $p$ is the characteristic of $G(q)$. Then $P=$ $N_{G}(V)$, and $P=L V$ (semidirect product), where $L=L_{J}(q)$ is a member of a family of finite groups of Lie type having the Weyl group $W_{J}$.

Note that this result explains why finite abelian groups of order prime to $p$ (which appear in the semidirect product decomposition of the Borel subgroup) were included in the definition of finite groups of Lie type.

The semidirect product $P=L V$ in (1.3.2) is called a Levi decomposition of $P$, and a subgroup $L$ (isomorphic to $\left.P / O_{p}(P)\right)$ a Levi factor of $P$.

The interesting group theoretic condition $P=N_{G}\left(O_{p}(P)\right)$ in (1.3.2) can be shown to characterize parabolic subgroups, (see [8]; the proof uses the theory of reductive algebraic groups).

Returning to our examples once again, the standard parabolic subgroups in $G L_{n}(q)$ are parametrized by partitions of $n, n=n_{1}+\cdots+n_{s}$, corresponding to parabolic subgroups of the Weyl group $S_{n}$ of the form

$$
S_{n_{1}} \times \cdots \times S_{n_{s}} \text {. }
$$

The corresponding standard parabolic subgroup consists of all partitioned matrices

$$
\left(\begin{array}{ccc}
A_{11} & A_{12} & \ldots \\
A_{21} & \cdot & \ldots \\
\cdot & \cdot & \cdot
\end{array}\right),
$$

where $A_{i j}$ is an $n_{i} \times n_{j}$ matrix, and $A_{i j}=0$ if $i>j$. The $O_{p}$-subgroup of such a parabolic subgroup consists of the matrices as above with $A_{i i}$ equal to the identity matrix $I_{n_{i}}$. A Levi factor is given by the partitioned matrices with $A_{i j}=0$ for $i \neq j$, and is isomorphic to

$$
G L_{n_{1}}(q) \times \cdots \times G L_{n_{s}}(q),
$$

a finite group of Lie type with Weyl group $S_{n_{1}} \times \cdots \times S_{n_{s}}$, as in (1.3.2).

2. Representations and characters. This section contains a review of basic principles of representation theory over the complex field $\mathbf{C}$. Special attention is given to the classical methods of constructing characters, by induction from subgroups (Frobenius) and by Brauer's criterion for virtual characters. The results on induced characters are combined with ideas in $\$ 1$ to describe Harish-Chandra's organization of the character theory of a finite group of Lie type, in terms of the philosophy of cusp forms.

2.1 Basic principles of representation theory. A representation of a finite group $G$ (over the complex field) is a homomorphism

$$
S: G \rightarrow G L(M)
$$

from $G$ into the group of invertible linear transformations on a finite-dimensional vector space $M$ over $\mathbf{C}$. The character $\chi$ of $S$ is the complex valued 
function $\chi: G \rightarrow \mathbf{C}$ defined by the trace map:

$$
\chi(g)=\operatorname{Tr}(g, S),
$$

where $\operatorname{Tr}(g, S)$ is the trace of the linear transformation $S(g)$. The degree of the character is the value at the identity element of $G$,

$$
\operatorname{deg} \chi=\chi(1),
$$

so that $\operatorname{deg} \chi=\operatorname{dim}_{\mathrm{C}}(M)$.

Two representations of $G, S: G \rightarrow G L(M)$ and $S^{\prime}: G \rightarrow G L\left(M^{\prime}\right)$, are equivalent if one is carried to the other by an isomorphism $X: M \rightarrow M^{\prime}$ of the underlying vector spaces:

$$
S(g)=X^{-1} S^{\prime}(g) X, \quad \forall g \in G .
$$

Two representations are equivalent if and only if they have the same character. Thus the problem of constructing the characters of $G$, while somewhat simpler than the problem of constructing the representations, nevertheless captures much of the representation-theoretic information.

A representation is irreducible if it cannot be expressed as a direct sum of representations on smaller vector spaces. Every representation is a direct sum of irreducible ones, uniquely determined up to equivalence, so the central problem of representation theory is to construct the irreducible representations and their characters (called the irreducible characters). All these concepts also apply to representations of semisimple associative algebras over $\mathbf{C}$.

There is a kind of Fourier analysis involving the characters of a finite group that is extremely useful and powerful. We note first that because of properties of the trace map, the characters $\chi$ are class functions, that is, they are constant on conjugacy classes: $\chi\left(x g x^{-1}\right)=\chi(g)$ for all $x, g \in G$. We next introduce a hermitian scalar product in the vector space of complex valued class functions $\operatorname{cf}(G)$ on $G$ :

$$
(\varphi, \psi)=|G|^{-1} \sum_{x \in G} \varphi(x) \overline{\psi(x)}, \quad \varphi, \psi \in \operatorname{cf}(G) .
$$

It then turns out that the irreducible characters $\left\{\chi_{i}\right\}$ form an orthonormal basis in the vector space of class functions $\operatorname{cf}(G):\left(\chi_{i}, \chi_{j}\right)=\delta_{i j}$ (Kronecker delta), and every class function $\chi$ can be expressed uniquely in terms of the irreducible characters: $\chi=\Sigma c_{i} \chi_{i}, c_{i} \in \mathbf{C}$. In particular, the character $\chi$ of an arbitrary representation $S$ can be expressed as a linear combination

$$
\chi=\sum n_{i} x_{i}
$$

with nonnegative integer coefficients $n_{i}$, called the multiplicities of $\left\{\chi_{i}\right\}$ in $\chi$. The multiplicities $n_{i}$ count the number of times the irreducible representation $S_{i}$ with character $\chi_{i}$ appears as a direct summand of the representation $S$.

The multiplicities $n_{i}$ in the expression (2.1.1) are computed from the inner product as $n_{i}=\left(\chi, \chi_{i}\right)$, and thus play a role analogous to Fourier coefficients. We shall sometimes write $\chi_{i} \in \chi$ to indicate that the multiplicity $\left(\chi_{i}, \chi\right)$ of the irreducible character $\chi_{i}$ in $\chi$ is positive, $\left(\chi_{i}, \chi\right)>0$.

For a character $\chi$ as in (2.1.1), we have for the norm $\|\chi\|$ of $\chi$,

$$
\|\chi\|^{2}=(\chi, \chi)=\sum n_{i}^{2}
$$


and consequently obtain a useful criterion for irreducibility: a character $\chi$ is irreducible if and only if $(\chi, \chi)=1$.

From the discussion above, it follows that the number of distinct irreducible characters is finite, and is less than or equal to the number of conjugacy classes in $G$; in fact, these two numbers are equal.

An important method of construction of characters of $G$ from characters of subgroups $H$ of $G$ was discovered by Frobenius. Let $\psi$ be a complex valued class function on $H$, and define a function $\psi^{G}$ on $G$ by the formula

$$
\psi^{G}(g)=|H|^{-1} \sum_{x \in G} \dot{\psi}\left(x g x^{-1}\right),
$$

where $\dot{\psi}$ is the function on $G$ obtained by letting $\dot{\psi}$ coincide with $\psi$ on $H$, and setting it equal to zero outside $H$. Then $\psi^{G}$ is a class function on $G$. Moreover, an easy calculation shows that we have:

(2.1.1) Proposition (Frobenius Reciprocity). For all $\psi \in \operatorname{cf}(H)$ and $\chi \in \operatorname{cf}(G)$ we have

$$
\left(\chi, \psi^{G}\right)_{G}=(\chi \mid H, \psi)_{H}
$$

where the scalar products are computed on the groups $G$ and $H$ respectively, and $\chi \mid H$ denotes the restriction of $\chi$ to the subgroup $H$.

Using Frobenius reciprocity, it is easily checked that, if $\psi$ is a character of $H$, then $\psi^{G}$ is a character of $G$. This simple observation is already one of the important methods for the construction of characters of $G$; however, even if $\psi$ is an irreducible character of $H, \psi^{G}$ need not be irreducible. For example, if $1_{H}$ is the trivial character on $H$ (such that $1_{H}(h)=1$ for all $h \in H$ ), then from Frobenius reciprocity we have

$$
\left(1_{H}^{G}, 1_{H}^{G}\right)=|H \backslash G / H|,
$$

the numbers of $(H, H)$-double cosets, so that if $H \neq G, 1_{H}^{G}$ is never irreducible. To make full use of Frobenius' construction, therefore, we must also consider the problem of decomposing induced characters $\psi^{G}$ into irreducible ones.

Considerable insight towards the decomposition problem comes from the following theorem of Mackey. We use the notation $H^{x}$ to denote the conjugate $x^{-1} H x$ of a subgroup $H$ of $G$ by an element $x \in G$. If $\psi$ is a character of $H$, then $\psi^{x}$ denotes the character of $H^{x}$ given by $\psi^{x}\left(h^{x}\right)=\psi(h)$, $\forall h \in H$.

(2.12) INTERTWINING NUMBeR THeOREM. Let $\psi_{1}$ and $\psi_{2}$ be characters of subgroups $H_{1}$ and $H_{2}$ of a finite group $G$. Then

$$
\left(\psi_{1}^{G}, \psi_{2}^{G}\right)=\sum_{H_{1} x H_{2}}\left(\psi_{1}^{x}, \psi_{2}\right)_{H_{1}^{x} \cap H_{2}}
$$

where the sum is taken over the distinct $\left(H_{1}, H_{2}\right)$-double cosets in $G$.

A second powerful method for constructing characters was discovered by Brauer. Let $\operatorname{char}_{Z}(G)$ denote the set of $Z$-linear combinations of the irreducible characters of $G$. The elements of $\operatorname{char}_{Z}(G)$, while not always characters 
themselves, can always be expressed as differences of two characters. Elements of $\operatorname{char}_{Z}(G)$ are called virtual characters (or generalized characters). Similarly we may define virtual representations of $G$. For a virtual representation $S$ with virtual character $\varphi$, we shall denote the value of $\varphi$ at an element of $g \in G$ by $\operatorname{Tr}(g, S)$, even though $\varphi(g)$ is not necessarily the trace of a linear transformation.

Brauer's theorem gives a test to determine whether a given class function is a virtual character. For the statement, we require the important concept of an elementary subgroup of $G$, which is a subgroup of the form $\langle g\rangle \times P$ (direct product) where $\langle g\rangle$ is a cyclic group, and $P$ is a $p$-group, for some prime $p$.

(2.1.3) BRAUER's THEOREM. A class function $\varphi$ on $G$ is a virtual character if and only if $\varphi \mid E$ is a virtual character of $E$, for all elementary subgroups $E$ of $G$.

Brauer's theorem can often be used to show that class functions on $G$ defined in some natural way, are in fact virtual characters. One can then attempt to decompose them into irreducible characters, noting that for a virtual character $\varphi,(\varphi, \varphi)=1$ if and only if $\pm \varphi$ is an irreducible character of G.

(2.2) The philosophy of cusp forms. The main problems. Throughout this section, let $G=G(q)$ denote a finite group of Lie type, of characteristic $p$, belonging to a family with a common Weyl group W (see \$1.3). As a start towards an inductive method of constructing the irreducible characters of $G$, let us assume for the time being that the characters of groups of Lie type of smaller rank than $G$, are known. We then ask what can be said about the irreducible characters of the groups $\{G(q)\}$ in the family under consideration. We emphasize that the problem is not simply to find the characters of an individual group $G(q)$, but to find them by methods which will apply to all groups in the family.

An obvious starting point is the examination of proper parabolic subgroups of $G$. For such a subgroup $P$, there is, by Theorem (1.3.2) a Levi decomposition $P=L V$, where $L=L(q)$ is a finite group of Lie type belonging to a family of groups of lower rank than $G$.

Let $S$ be an irreducible representation of $L$ and let $\tilde{S}$ be the irreducible representation of $P$ obtained by lifting $S$ to $P$, putting $V=O_{p}(P)$ in the kernel. In other words, because of the semidirect product decomposition $P=L V$, there exists a homomorphism $f: P \rightarrow L$ with kernel $V$, and $\tilde{S}$ is simply the composite map $S \circ f$. Letting $\psi$ and $\tilde{\psi}$ denote the characters of $S$ and $\tilde{S}$, we see by Mackey's theorem (2.1.2) that

$$
\left(\tilde{\psi}^{G}, \tilde{\psi}^{G}\right)=\sum_{P x P}\left(\tilde{\psi}^{x}, \tilde{\psi}\right)_{P^{x} \cap P}
$$

If $P$ is a standard parabolic subgroup $B W_{J} B$, it follows from (1.3.1) that the double cosets $P x P$ are in bijective correspondence with the double cosets $W_{J} \backslash W / W_{J}$, and hence, to some extent, the norm $\left\|\tilde{\psi}^{G(q)}\right\|^{2}$ is independent of $q$. In contrast, $\left\|\psi^{G(q)}\right\|^{2}$ involves the double cosets $L(q) \backslash G(q) / L(q)$, which depend on $q$ in an uncontrollable way.

The irreducible characters $\chi$ appearing with positive multiplicity in $\tilde{\psi}^{G}$ will 
satisfy, by Frobenius reciprocity,

$$
\left(\chi, 1_{V}^{G}\right) \neq 0
$$

where $V$ is the $O_{p}$-subgroup of some proper parabolic subgroup of $G$.

Harish-Chandra formulated a general principle, which he called the philosophy of cusp forms, which applies to the above situation in the theory of finite groups of Lie type, and also to the representation theory of real semisimple Lie groups, and reductive algebraic groups over $p$-adic fields.

In our situation, the main ideas can be described as follows.

(2.2.2) Definition. An irreducible character $\chi$ of $G$ is said to be cuspidal (or belongs to the discrete series) if $\left(\chi, 1_{V}^{G}\right)=0$ for all $O_{p}$-subgroups $V$ of proper parabolic subgroups $P$ of $G$.

In other words the cuspidal characters are those that are missed entirely by the inductive considerations at the beginning of this section. (See (2.2.1).)

(2.2.3) TheOREM (HARISH-ChANDRA). Let $G(q)$ be a member of a family of finite groups of Lie type with Weyl group $W$. Then:

(i) Every irreducible character $\chi$ of $G(q)$ is either cuspidal or $\left(\chi, \tilde{\psi}^{G(q)}\right) \neq 0$ for some irreducible cuspidal character $\psi$ of a Levi factor $L(q)$ of some proper parabolic subgroup $P(q)$ of $G(q)$.

(ii) If the second alternative occurs, then $P(q)$ and $\psi$ are essentially uniquely determined by $\chi$. More precisely, if $\psi$ and $\psi^{\prime}$ are irreducible cuspidal characters of Levi factors $L(q)$ and $L^{\prime}(q)$ of proper parabolic subgroups $P(q)$ and $P^{\prime}(q)$ of $G$, then

$$
\left(\tilde{\psi}^{G(q)}, \tilde{\psi}^{\prime G(q)}\right)=0
$$

unless $L(q)$ is conjugate to $L^{\prime}(q)$ in $G(q)$ and $\psi$ and $\psi^{\prime}$ are conjugate characters of $L(q)$ and $L^{\prime}(q)$.

(iii) Let $\psi$ be an irreducible cuspidal character of a Levi factor $L(q)$, as in (i). Then $\left(\tilde{\psi}^{G(q)}, \tilde{\psi}^{G(q)}\right)$ is bounded independently of $q$; in fact

$$
\left(\tilde{\psi}^{G(q)}, \tilde{\psi}^{G(q)}\right) \leqslant|W| \text {. }
$$

Let us define two parabolic subgroups to be associated if their Levi factors are conjugate in $G(q)$. Statement (ii) asserts that the organization of the characters of $G(q)$ according to (2.2.3) is as efficient as possible. That is, each irreducible noncuspidal character $\chi$ of $G(q)$ appears in $\tilde{\psi}^{G(q)}$ for a uniquely determined conjugacy class of irreducible cuspidal characters of Levi factors of a uniquely determined equivalence class of associated parabolic subgroups. Thus the following problems (neither of which is completely solved at this time) are fundamental for the determination of the irreducible characters of $G(q)$ :

(A) (CONSTRUCTION OF THE DISCRETE SERIES). To construct the irreducible cuspidal characters of an arbitrary finite group $G$ of Lie type; and

(B) (Decomposition Problem). To decompose $\tilde{\psi}^{G}$ into irreducible components, for an irreducible cuspidal character $\psi$ of a Levi factor of a proper parabolic subgroup of $G$.

3. Decomposition of induced characters. This section begins with some general principles governing the decomposition of induced characters for 
arbitrary finite groups. The methods are applied to finite groups of Lie type in $\$ 3.2$, and in particular, to the simplest case of the decomposition problem, stated in $\$ 2.2$.

(3.1) Centralizers and Hecke algebras. Let $H$ be a subgroup of a finite group $G$, and let $\psi$ be an irreducible character of $H$. There exists a primitive idempotent $e_{\psi}$ in the complex group algebra $\mathrm{CH}$ such that $\psi$ is the trace function associated with the action of $H$ by left multiplication on the minimal left ideal $\mathbf{C H e} e_{\psi}$. Let us view $\mathrm{CH}$ as a subalgebra of $\mathrm{CG}$. It is not difficult to prove that the character of $G$ afforded by the left $\mathrm{C} G$-module $\mathrm{CGe}_{\psi}$ is the induced character $\psi^{G}$.

Definition. The centralizer of the left $\mathrm{CG}$-module $\mathrm{CGe}_{\psi}$ (or the centralizer of the representation of $G$ by left multiplication on $\mathrm{CGe}_{\psi}$ ) is defined to be the algebra of linear transformations $\mathrm{End}_{\mathbf{C} G}\left(\mathrm{CGe}_{\psi}\right)$, consisting of all linear transformations on the vector space $\mathrm{CGe}_{\psi}$ which commute with the action of $G$.

An easy lemma from ring theory shows that $\operatorname{End}_{\mathbf{C} G}\left(\mathrm{CGe}_{\psi}\right)$ is antiisomorphic to $e_{\psi} \mathbf{C} G e_{\psi}$, which is a semisimple subalgebra of $\mathbf{C} G$ with identity element $e_{\psi}$. Schur's Lemma implies that the induced character $\psi^{G}$ is irreducible if and only if $\operatorname{dim}_{\mathrm{C}}\left(e_{\psi} \mathrm{CG} e_{\psi}\right)=1$. The structure of $e_{\psi} \mathbf{C G} e_{\psi}$ yields information about the decomposition of $\psi^{G}$ in the general case.

For example, the idempotent $e_{H}=|H|^{-1} \Sigma_{h \in H} h$ affords the trivial representation $1_{H}$ of $H$, and hence $\mathrm{CGe}_{H}$ affords the induced permutation character $1_{H}^{G}$. Recalling that the group algebra $\mathbf{C} G$ may be identified with all complex valued functions on $G$, multiplied by convolution

$$
(f g)(x)=\sum_{y \in G} f(x y) g\left(y^{-1}\right), \quad f, g \in \mathbf{C} G, x \in G,
$$

we see that the algebra $e_{H} \mathrm{CGe} e_{H}$ is the subalgebra of $\mathrm{C} G$ consisting of all functions which are constant on $(H, H)$-double cosets (multiplied by convolution). Closely related algebras and their representations constitute the theory of spherical functions in the representation theory of Lie groups, and what follows may be viewed as the counterpart, in finite group theory, of some aspects of the theory of spherical functions.

DEFINITION. Let $H$ be a subgroup of $G$, and $\psi$ a character of $H$ afforded by a left ideal $\mathrm{CHe} e_{\psi}$ generated by an idempotent $e_{\psi} \in \mathrm{CH}$. The Hecke algebra $H(G, H, \psi)$ is the subalgebra $e_{\psi} \mathrm{CGe} e_{\psi}$ of $\mathrm{C} G$.

(3.1.1) Theorem. Let $G, H, \psi, H(G, H, \psi)$ be as above. Let $\chi$ be an irreducible character of $G$, extended by linearity to a function on the group algebra $\mathbf{C} G$. Then $\left(\chi, \psi^{G}\right) \neq 0$ if and only if the restriction $\left.\chi\right|_{H(G, H, \psi)} \neq 0$. In case the restriction is not zero, it defines an irreducible character $\omega_{\chi}$ of the Hecke algebra of degree $\left(\chi, \psi^{G}\right)$. Moreover, the map $\left.\chi \rightarrow \chi\right|_{H(G, H, \psi)}$ defines a bijection from the irreducible components $\{\chi\}$ of $\psi^{G}$ to the set of all irreducible characters of the Hecke algebra $H(G, H, \psi)$.

The next result shows that the character values of the irreducible components of $\psi^{G}$ can be computed from a knowledge of the representations of the Hecke algebra.

(3.1.2) TheORem (ReE). Let $G, H, \psi, H(G, H, \psi)$ be as above, and let $\chi$ be 
an irreducible component of $\psi^{G}$. Let $\omega_{\chi}$ be the irreducible character of $H(G, H, \psi)$ given by $\left.\chi\right|_{H(G, H, \psi)}$. Let $t \in G$, let $\mathbb{E}$ be the conjugacy class of $t$, and $C=\Sigma_{x \in \Subset} x$ the class sum, and let $Z_{G}(t)$ denote the centralizer of $t$ in $G$. Then

$$
\chi(t)=\frac{\left|Z_{G}(t)\right| \omega_{\chi}(e C e)}{\Sigma_{g \in G} \omega_{\chi}\left(e g^{-1} e\right) \omega_{\chi}(e g e)} .
$$

Computation of $\chi(t)$ from this formula is not easy. The result becomes considerably simplified in case $\psi$ is a linear character of $H$. We illustrate in the case of permutation representations $1_{H}^{G}$.

For $H$ a subgroup of $G$, and $x \in G$, let

$$
\text { ind }_{H} x=\left|H: H \cap H^{x}\right|
$$

then ind $_{H} x$ is the number of $H$-cosets in the double coset $H x H$.

(3.1.3) Theorem. Let $H$ be a subgroup of $G$, and let $H\left(G, H, 1_{H}\right)$ be the Hecke algebra corresponding to the trivial representation of $H$. Let $\left\{D_{i}\right\}_{i \in I}$ denote the $(H, H)$-double cosets in $G$. The normalized characteristic functions

$$
a_{i}=\frac{1}{|H|} \sum_{x \in D_{i}} x, \quad i \in I,
$$

form a basis of $H\left(G, H, 1_{H}\right)$, for which the structure constants are rational integers, and are given by

$$
a_{i} a_{j}=\sum \mu_{i j k} a_{k}
$$

with

$$
\mu_{i j k}=|H|^{-1}\left|D_{i} \cap x_{k} D_{j}^{-1}\right|, \text { for } x_{k} \in D_{k} .
$$

Letting $\hat{a}_{i}$ denote the basis element corresponding to the double coset $D_{i}^{-1}$, the character formula (3.1.2), for $\chi \in 1_{H}^{G}$, and $t \in G$, becomes

$$
\chi(t)=\frac{\left|Z_{G}(t)\right|}{|H|} \frac{\sum\left(\text { ind } x_{i}\right)^{-1} \omega_{\chi}\left(a_{i}\right)\left|\mathfrak{L} \cap D_{i}\right|}{\sum\left(\text { ind } x_{i}\right)^{-1} \omega_{\chi}\left(\hat{a}_{i}\right) \omega_{\chi}\left(a_{i}\right)},
$$

where the sum is taken over double cosets, and $x_{i} \in D_{i}$ for $i \in I$. In particular,

$$
\operatorname{deg} \chi=\frac{|G: H| \operatorname{deg} \omega_{\chi}}{\sum\left(\operatorname{ind} x_{i}\right)^{-1} \omega_{\chi}\left(\hat{a}_{i}\right) \omega_{\chi}\left(a_{i}\right)},
$$

where $\operatorname{deg} \omega_{\chi}=\left(\chi, \psi^{G}\right)$.

(3.2) Principal series representations. Generic degrees. We shall consider the application of Hecke algebras to the simplest case of the decomposition problem, namely the description of the irreducible components of $\tilde{\lambda}^{G}$, for a cuspidal character $\lambda$ of a Levi factor $L$ of a parabolic subgroup $P$ of a finite group of Lie type $G$, in the minimal situation, with $P$ a Borel subgroup. In this case, the Levi factor $T$ is an abelian group of order prime to the characteristic, and has no proper parabolic subgroups, so that all irreducible characters of $T$ are, by definition, cuspidal. The components of $\tilde{\lambda}^{G}$ in this situation are called principal series characters of $G$, in analogy with repre- 
sentations of semisimple Lie groups associated with minimal parabolic subgroups. The principal series characters appearing in $\tilde{\lambda}^{G}$, as $\lambda$ ranges over the linear characters of $T$, are simply the components of $1_{U}^{G}$ where $U$ is the $O_{p}$-subgroup of a Borel subgroup.

The problem of decomposing $\tilde{\lambda}^{G}$, for $\lambda$ an arbitrary linear character of $T$ lifted to the Borel subgroup of $B$, is not essentially more difficult than the analysis of $1_{B}^{G}$, where $1_{B}$ is the trivial character of $B$. Therefore we shall begin with the case of $1_{B}^{G}$, and will discover later that the methods used in this situation are of broad applicability to other cases of the decomposition problem.

From $\S 3.1$, the Hecke algebra $H\left(G, B, 1_{B}\right)$ associated with $1_{B}^{G}$, has a basis consisting of the normalized characteristic functions on the $(B, B)$-double cosets, which, by the Bruhat Theorem 1.2.1, are parametrized by the elements of the Weyl group $W$. Therefore the basis elements of $H\left(G, B, 1_{B}\right)$ are

$$
a_{w}=\frac{1}{|B|} \sum_{x \in B w B} x, \quad w \in W .
$$

For the next result, we require the concept of the length $l(w)$ of an element of a finite Coxeter group $W$, defined as the minimal number of factors among all expressions of $w$ as a product of the distinguished generators.

(3.2.1) Theorem (IWAhori, Matsumoto). The multiplication in $H\left(G, B, 1_{B}\right)$, for a finite group $G$ with a $(B, N)$-pair, satisfies

$$
a_{w_{i}} a_{w}=\left\{\begin{array}{l}
a_{w_{i} w}, \quad l\left(w_{i} w\right) \geqslant l(w), \\
q_{i} a_{w_{i} w}+\left(q_{i}-1\right) a_{w}, \quad l\left(w_{i} w\right)<l(w),
\end{array}\right.
$$

for all $w \in W$ and distinguished generators $\left\{w_{i}\right\}, 1 \leqslant i \leqslant n$, where $q_{i}=$ $\operatorname{ind}_{B} w_{i}\left(=\left|B: B \cap B^{w_{1}}\right|\right)$. Moreover, $H\left(G, B, 1_{B}\right)$ has a presentation as an associative algebra with generators $\left\{a_{w_{i}}\right\}_{1 \leqslant i \leqslant n}$ and relations as follows:

(quadratic relations) $a_{w_{i}}^{2}=q_{i} 1+\left(q_{i}-1\right) a_{w_{i}}$, and

(homogeneous relations) $a_{w_{i}} a_{w_{j}} a_{w_{i}} \cdots=a_{w_{j}} a_{w_{i}} a_{w_{j}} \cdots(i \neq j)$

with $n_{i j}$ factors, where $n_{i j}$ is the order of the product $w_{i} w_{j}$ in $W$.

For example, in case $W=S_{3}$, the generators of $H\left(G, B, 1_{B}\right)$ are $\left\{a_{w_{1}}, a_{w_{2}}\right\}$, with relations

$$
a_{w_{i}}^{2}=q_{i} 1+\left(q_{i}-1\right) a_{w_{i}}, \quad i=1,2
$$

and

$$
a_{w_{1}} a_{w_{2}} a_{w_{1}}=a_{w_{2}} a_{w_{1}} a_{w_{2}} \text {. }
$$

Using the presentation in (3.2.1), it is immediate that the Hecke algebra $H\left(G, B, 1_{B}\right)$ always has two one-dimensional representations, ind and sgn, defined on the generators as follows:

$$
\operatorname{ind}\left(a_{w_{i}}\right)=q_{i}, \quad 1 \leqslant i \leqslant n,
$$

and

$$
\operatorname{sgn}\left(a_{w_{i}}\right)=-1, \quad 1 \leqslant i \leqslant n .
$$


The corresponding representations in $1_{B}^{G}$ according to Theorem 3.1.1 are: the trivial representation $1_{G}$, corresponding to ind; and the Steinberg representation $\mathrm{St}_{G}$, corresponding to sgn. As an exercise the reader can show, using the degree formula in (3.1.3) and some facts about groups with split $(B, N)$-pairs, that

$$
\operatorname{deg} \mathrm{St}_{G}=|G|_{p}
$$

where $|G|_{p}$ denotes the $p$-part of the order of $G$.

Using the homomorphism ind, and the homogeneous relations in $H\left(G, B, 1_{B}\right)$, it follows that the index parameters $\left\{q_{i}\right\}$ satisfy the condition $q_{i}=q_{j}$ whenever $w_{i}$ and $w_{j}$ are conjugate in $W$.

If we set the index parameters $q_{i}=1$, we see that the Hecke algebra becomes the group algebra $\mathbf{C W}$ of $W$. The connection between these algebras can be investigated, following Tits, by introducing the generic algebra $A_{W}$ of the finite Coxeter group $W$, as follows. Let $R=Q\left[X_{1}, \ldots, X_{n}\right]$ denote the polynomial ring over the rational field $Q$ with generators $\left\{X_{i}\right\}$ corresponding to the distinguished generators $\left\{w_{i}\right\}$ of $W$, and satisfying the condition that $X_{i}=X_{j}$ whenever $w_{i}$ and $w_{j}$ are conjugate in $W$. (For example, if $W$ is an indecomposable Coxeter group, it follows that $R$ is a polynomial ring in either one or two indeterminates.)

Definition. The generic algebra $A_{W}$ is the algebra over $R$, with basis $\left\{\alpha_{w}\right\}_{w \in W}$ over $R$, identity element $\alpha_{1}$, and multiplication defined by the conditions

$$
\alpha_{w_{i}} \alpha_{w}=\left\{\begin{array}{l}
\alpha_{w_{i} w}, \quad l\left(w_{i} w\right) \geqslant l(w), \\
X_{i} \alpha_{w_{i} w}+\left(X_{i}-1\right) \alpha_{w}, \quad l\left(w_{i} w\right) \leqslant l(w)
\end{array}\right.
$$

The structure constants of the generic algebra are polynomials in $X_{1}, \ldots, X_{n}$. A homomorphism $f: R \rightarrow Q$ is defined by specialization, i.e. substitution of elements $q_{i} \in Q$ for the variables $X_{i}$, so that $f\left(P\left(X_{1}, \ldots, X_{n}\right)\right)$ $=P\left(q_{1}, \ldots, q_{n}\right)$ for all $P\left(X_{1}, \ldots, X_{n}\right) \in R$. For each such specialization $f$ : $R \rightarrow Q$, we obtain an algebra over $Q$, called the specialized algebra $A_{f}$, whose structure constants are obtained by applying $f$ to the structure constants of the generic algebra. In particular it is clear from (3.2.1) that if $G(q)$ is a member of a system of finite groups of Lie type, with Weyl group $W$ and index parameters $\left\{q_{i}, 1 \leqslant i \leqslant n\right\}$, then the specialized algebra $A_{f}$, for the specialization $f: R \rightarrow Q$ given by $f\left(X_{i}\right)=q_{i}$, is isomorphic to the Hecke algebra $H\left(G(q), B(q), 1_{B(q)}\right)$, while the specialized algebra $A_{f_{1}}$ given by $f_{1}\left(X_{i}\right)$ $=1,1 \leqslant i \leqslant n$, is isomorphic to the group algebra $Q W$.

From these facts, the associativity of $A_{W}$ follows, because the associativity is equivalent to a polynomial relation in $\left\{X_{1}, \ldots, X_{n}\right\}$ which is satisfied by all the specializations $X_{i} \rightarrow q_{i}$ arising from groups in the system under consideration. Similarly, the discriminant $\delta$ of the algebra $A_{W}$ is a polynomial in $\left\{X_{1}, \ldots, X_{n}\right\}$ whose image under the specializations $X_{i} \rightarrow q_{i}$ is different from zero, hence $\delta \neq 0$. Letting $K$ denote the quotient field of $R$, it follows that the algebra $A_{W}^{K}=K \otimes_{R} A_{W}$ is a separable algebra over $K$, that is, $A_{W}^{K}$ is semisimple, and remains semisimple over all extension fields of $K$.

As in the case of $H\left(G, B, 1_{B}\right)$, it follows that $A_{W}$ has a presentation exactly 
as in (3.2.1), with quadratic relations

$$
\alpha_{w_{i}}^{2}=X_{i} 1+\left(X_{i}-1\right) \alpha_{w_{i}}, \quad 1 \leqslant i \leqslant n,
$$

and the same homogeneous relations. It then follows, as before, that $A_{W}$ has two homomorphisms into $R$, IND and SGN, defined by

$$
\operatorname{IND}\left(\alpha_{w_{i}}\right)=X_{i} \text { and } \operatorname{SGN}\left(\alpha_{w_{i}}\right)=-1, \quad 1 \leqslant i \leqslant n \text {. }
$$

Using the generic algebra as an intermediary, the structures of specialized algebras $A_{f}$ and $\boldsymbol{A}_{f^{\prime}}$ for different specializations $f$ and $f^{\prime}$, can be compared.

(3.2.2) Deformation TheOREM (TITs). Let $f$ and $f^{\prime}$ be homomorphisms from $R \rightarrow Q$ such that the specialized algebras $A_{f}$ and $A_{f^{\prime}}$ are semisimple. Then the algebras $A_{f}^{\mathbf{C}}$ and $A_{f^{\prime}}^{\mathbf{C}}$,obtained by extension of the field to $\mathbf{C}$, are semisimple and isomorphic.

(3.2.3) COROLlaRY. Let $G(q)$ be a member of a family of finite groups of LIe type with Weyl group $W$. Then there exists an isomorphism

$$
H\left(G(q), B(q), 1_{B(q)}\right) \cong \mathbf{C W} .
$$

The isomorphism above is not given canonically. For applications to the representation theory of $G(q)$, it is desirable to have a more precise comparison between the representations of $H\left(G(q), B(q), 1_{B(q)}\right)$ and $\mathrm{CW}$, which can be achieved as follows.

(3.2.4) Proposition. Let $A_{W}$ be a generic algebra over $R$, let $K$ be the quotient field of $R$, let $\bar{K}$ be an algebraic closure of $K$, and $\bar{R}$ the integral closure of $R$ in $\bar{K}$. Let $f: R \rightarrow Q$ be a homomorphism such that the specialized algebra $A_{f}$ is semisimple, and let $\bar{f}: \bar{R} \rightarrow \mathbf{C}$ be a fixed extension of $f$ to a homomorphism from $\bar{R}$ into the algebraic closure of $Q$ in $\mathbf{C}$. Let $\chi$ be an irreducible character of the semisimple algebra $A_{W}^{\bar{K}}$. Then $\chi\left(\alpha_{w}\right) \in \bar{R}$ for each basis element $\alpha_{w}, w \in W$. Moreover, letting $f\left(\alpha_{w}\right)$ denote the basis element of $A_{f}$ corresponding to $\alpha_{w}$, the map $\chi_{\bar{f}}: A_{f}^{\mathbf{C}} \rightarrow \mathbf{C}$ defined by $\chi_{f}\left(f\left(\alpha_{w}\right) \otimes 1\right)=\bar{f}\left(\chi\left(\alpha_{w}\right)\right)$, extended by linearity to a linear map on the semisimple algebra $A_{f}^{\mathbf{C}}$, is an irreducible character of $A_{f}^{\mathbf{C}}$. The correspondence $\chi \rightarrow \chi_{\bar{f}}$ is a bijection from the irreducible characters of $A_{W}$ to the irreducible characters of $A_{f}^{\mathbf{C}}$.

The correspondence $\chi \rightarrow \chi_{\bar{f}}$ depends in general on the choice of the extension $\bar{f}$ of $f: R \rightarrow Q$. The correspondence will be independent of $\bar{f}$ provided that the characters of the generic algebra $A_{W}^{\bar{K}}$ are rational, in the sense that $\chi\left(\alpha_{w}\right) \in R$ for all $w \in W$, for each irreducible character $\chi$ of $A_{W}^{\bar{K}}$. Rationality holds most of the time, but there are some interesting exceptions.

(3.2.5) THEOREM (BENSON AND CURTIS). Let $A_{W}$ be a generic algebra of an indecomposable Coxeter group $W$ associated with a family of finite groups of Lie type. Then every irreducible character of $A_{W}^{\bar{K}}$ is rational, with the exception of the two irreducible characters of degree 512 in type $E_{7}$, the four irreducible characters of degree 4096 in type $E_{8}$, and the three nonlinear irreducible characters in case $W$ is dihedral of order 16 (corresponding to the family of twisted groups of type $F_{4}$ ).

A further result in this direction can be proved, namely that not only the 
characters, but the irreducible representations of the algebras $A_{W}^{\bar{K}}$, can be realized in the field $K$, with the exceptions noted above. From this it follows that, in cases where the above exceptions do not occur, the rational Hecke algebras $e_{B} Q G(q) e_{B}$ and the rational group algebras $Q W$ are isomorphic.

We conclude this section by showing how the preceding results can be combined with the theory of Hecke algebras to determine the degrees of the irreducible characters in $1_{B}^{G}$; further results on explicit calculation of character values are described in $\$ 4$.

Definition. Let $A_{W}$ be a generic algebra, and $\chi$ an irreducible character of $A_{W}^{\bar{K}}$. The generic degree corresponding to $\chi$ is the element of $\bar{K}$ given by

$$
d_{\chi}=\frac{(\operatorname{deg} \chi) P(X)}{\sum_{w \in W}\left(\operatorname{IND}\left(\alpha_{w}\right)\right)^{-1} \chi\left(\alpha_{w}\right) \chi\left(\alpha_{w-1}\right)},
$$

where $P(X)$ is the Poincaré polynomial of $W$ and is defined by

$$
P(X)=\sum_{w \in W} \operatorname{IND}\left(\alpha_{w}\right)
$$

Let $f: X_{i} \rightarrow q_{i}$ be a specialization associated with a finite group $G(q)$ in a family with Weyl group $W$. Then $f(P(X))$ is the index $|G(q): B(q)|$, where $B(q)$ is a Borel subgroup of $G(q)$. Moreover, the character $\chi$ defines, by (3.2.4), an irreducible character of the specialized algebra $A_{f}^{\mathbf{C}}$ (which is isomorphic to the Hecke algebra $H\left(G(q), B(q), 1_{B}(q)\right)$ ). By (3.1.1), we may therefore associate with $\chi$ an irreducible character $\zeta_{\chi \bar{f}} \in 1_{B(q)}^{G(q)}$ (depending on an extension of $f$ to a homomorphism $\bar{f}: \bar{R} \rightarrow \mathbf{C}$ as in (3.2.4)). It then follows from the degree formula in (3.1.3), that

$$
\operatorname{deg} \zeta_{\chi \bar{f}}=\bar{f}\left(d_{\chi}\right)
$$

where $d_{\chi}$ is the generic degree associated with $\chi$. The generic degrees $d_{\chi}$ in fact belong to $R=Q\left[X_{1}, \ldots, X_{n}\right]$, for all indecomposable Weyl groups $W$, whenever the characteristic powers $\{q\}$ of the family includes all prime numbers. In one case where this does not occur (for the Ree groups of twisted type $F_{4}$ ), however, the generic degrees associated with the nonlinear characters of $W$ do not belong to $R$. In the Suzuki groups and Ree groups of twisted type $B_{2}$ and $G_{2}$, the only nontrivial character of the generic algebra is $\mathrm{SGN}$, and the corresponding generic degree is in $R$. The following result describes the generic degrees for all families of finite groups of Lie type other than the Suzuki and Ree groups.

(3.2.6) THEOREM. Let $\{G(q)\}$ be a system of finite groups of Lie type with indecomposable Weyl group $W$, with $W$ not dihedral of order 16. The index parameters $\left\{q_{i}\right\}$ of $G(q)$ are given by $q^{c_{i}}$, for some positive integers $\left\{c_{1}, \ldots, c_{n}\right\}$. For each $q$, there is a bijection $\varphi \leftrightarrow \zeta_{\varphi, q}$ between the irreducible characters $\{\varphi\}$ of $W$ and the irreducible components $\zeta_{\phi, q} \in 1_{B(q)}^{G(q)}$, which is canonical except for the characters of $W\left(E_{7}\right)$ and $W\left(E_{8}\right)$ listed in (3.2.5). For each irreducible character $\varphi$ of $W$, there exists a polynomial $d_{\varphi}(X) \in Q[X]$, 
with the following properties:

(i) $\operatorname{deg} \zeta_{\varphi, q}=d_{\varphi}(q)$;

(ii) $d_{\varphi}(1)=\operatorname{deg} \varphi=\left(\zeta_{\varphi, q}, 1_{B(q)}^{G(q)}\right)$;

(iii) $d_{\varphi}(X)=d_{\chi}\left(X^{c_{1}}, \ldots, X^{c_{n}}\right)$ where $d_{\chi}\left(X_{1}, \ldots, X_{n}\right) \in R$ is the generic degree associated with $\varphi$.

The fact that the generic degrees $d_{\varphi}(X)$ are polynomials leads immediately to information about their explicit form. For simplicity we restrict ourselves to the case of a family $\{G(q)\}$ of untwisted Chevalley groups. Then there is a polynomial $h(X) \in Q[X]$, called the group order polynomial, such that $|G(q)|$ divides $h(q)$ for all characteristic powers $q$. The group order polynomial has the form

$$
h(X)=X^{N}(X-1)^{n} P(X),
$$

where $N$ is the member of positive roots in the root system $\Phi$ associated with $G(q)$ (see $\S 1.1$ ), $n$ is the rank of the $(B, N)$-pair in $G(q)$, and $P(X)$ is the Poincare polynomial $\Sigma_{w \in W} X^{l(w)}$ of $W$ (see the definition of generic degree for a formula for $P(X)$ in the general case). Moreover, by a result of Chevalley, $P(X)$ can be expressed in multiplicative form

$$
P(X)=\prod_{i=1}^{n}\left(1+X+X^{2}+\cdots+X^{m_{i}}\right)
$$

where the $\left\{m_{i}\right\}$ are certain positive integers called the exponents of $W$, which are equal to the degrees of the basic polynomial invariants of $W$. For example, in the case of $S L_{n+1}(q)$, the basic polynomial invariants are the elementary symmetric functions, so that the exponents are $1,2, \ldots, n$. In any case, $P(X)$ can be expressed as a product $P(X)=\Pi \Phi_{i}(X)^{e_{i}}$ of explicitly known powers of cyclotomic polynomials $\left\{\Phi_{i}(X)\right\}$.

Now let $d_{\varphi}(X)$ be a generic degree, for some irreducible character $\varphi$ of $W$. Then by (3.2.6) and a basic result of character theory, we have $d_{\varphi}(q)|| G(q) \mid$ for all characteristic powers $q$. Because the set of characteristic powers is infinite, it follows that $d_{\varphi}(X) \mid h(X)$ in $Q[X]$, and hence, from unique factorization in $Q[X]$, we have

$$
d_{\varphi}(X)=\alpha_{\varphi} X^{k_{\varphi}} \prod \Phi_{i}(X)^{d_{i}}, \quad \alpha_{\varphi} \in Q,
$$

where the product is taken over cyclotomic polynomials $\left\{\Phi_{i}(X)\right\}$ different from $X-1$ (because $d_{\varphi}(1)=\varphi(1) \neq 0$ by (3.2.6)).

Using this information it can be proved that if $\varphi \neq 1_{W}$, then the exponent $k_{\varphi}>0$ in the above formula, and hence the degrees of all the characters $\zeta_{\varphi, q} \in 1_{B(q)}^{G(q)}$ are divisible by the characteristic $p$, with certain possible exceptions for primes dividing the denominator of $\alpha_{\varphi}$.

We now give a brief illustration of how this information can be applied to the group-theoretic problem of classifying the doubly transitive permutation representations of finite groups of Lie type $G$ with indecomposable Weyl groups. Let $\theta$ be the permutation character of such a permutation representation. Then $\theta=1_{H}^{G}$ for some subgroup $H$, and double transitivity means that $(\theta, \theta)=2$, so that $\theta=1_{G}+\chi$ for some irreducible character $\chi$. There is no a priori reason to expect $\chi$ to have anything to do with the characters in $1_{B}^{G}$. 
Nevertheless, if $\chi \notin 1_{B}^{G}$, then $\left(1_{B}^{G}, \theta\right)=1$, and we have $G=B H$. The possibilities for such a subgroup $H$ have been explicitly classified by Seitz [60]. So we may assume that $\chi \in 1_{B}^{G}$. Again by the main theorem of [60], we may assume $\theta$ is known if the characteristic $p$ divides $\operatorname{deg} \chi$. We are then reduced to the case $\chi \in 1_{B}^{G}$ and $p \nmid \operatorname{deg} \chi$. From the results on generic degrees, this leaves only a small number of possibilities for the characteristic $p$, and these can be discussed individually (see [22]).

Finally, let us return to the case of arbitrary principal series representations, for a family of adjoint Chevalley groups $G(q)$. In this situation, the stabilizer $W_{\lambda}$ of a linear character $\lambda$ of $T(q)$ in the Weyl group $W$, is a finite group generated by reflections, hence a Coxeter group. Moreover, it can be proved in this case that the Hecke algebra $H(G(q), B(q), \tilde{\lambda})$ can be obtained by specialization from the generic algebra $A_{W_{\lambda}}$. It follows that there is a bijection between the irreducible characters $\{\varphi\}$ of $W_{\lambda}$ and the irreducible components $\left\{\zeta_{\varphi, q}\right\}$ of $\tilde{\lambda}^{G}$, such that $\operatorname{deg} \zeta_{\varphi, q}$ is given by a generic formula involving the generic degrees $d_{\varphi}(X)$ of Theorem 3.2.6.

The generic degrees have now been computed for all indecomposable Coxeter groups (except for some characters of a generic ring of type $F_{4}$ over the polynomial ring in two variables). In the case of the classical groups, this involved carrying over to the generic rings classical results of Frobenius and Young on the characters of the symmetric and hyper-octahedral groups. In the case of the exceptional groups, the analysis of the characters of the generic ring was based on the determination of the character tables of $F_{4}$ by Kondo, and $E_{6}, E_{7}$ and $E_{8}$ by Frame.

4. Construction of cuspidal characters. The construction of characters other than those in the principal series depends, as we have shown in $\$ 2.2$, on the determination of the irreducible cuspidal characters (the discrete series). In their work on $G L_{n}(q), S p_{4}(q)$, and $G_{2}(q)$, Green, Srinivasan and Chang and Ree all used Brauer's Theorem 2.1.3 to construct virtual characters containing cuspidal characters as irreducible components. It had been recognized for some time that in order to make full use of this construction, more detailed information about the structure of finite groups of Lie type is needed, especially concerning conjugacy classes and centralizers of elements, then is available in the axiomatic framework of groups with split $(B, N)$-pairs. The framework for this set of questions is provided by the theory of reductive algebraic groups defined over finite fields. We begin this section with an introduction to this point of view, and then show what can be done in the new setting towards solving the main problems described in $\$ 2$.

4.1 Reductive groups over finite fields. Let $X$ be an affine algebraic variety, over a field $K$ which is the algebraic closure of a finite field $F_{p}$, and let $A$ denote the affine coordinate ring of $X$. For a finite field $F_{q} \subset K$, an $F_{q}-r a-$ tional structure on $X$ is an $F_{q}$-subalgebra $A_{0} \subset A$ such that $A_{0} \otimes_{F_{q}} K=A$. Given such a subalgebra $A_{0}$, we define $F: A \rightarrow A$ by $F\left(a_{0} \otimes \lambda\right)=a_{0}^{q} \otimes \lambda$, $\lambda \in K, a_{0} \in A_{0}$. Then $F$ is an algebra homomorphism, and is a bijection from $A \rightarrow A^{q}$, where $A^{q}=\left\{a^{q}: a \in A\right\}$. For each $a \in A$, there is an $n \geqslant 1$ such that $F^{n} a=a^{q^{n}}$. Conversely given such a homomorphism $F$, we can recover $A_{0}$ as the set $\left\{a \in A: F(a)=a^{q}\right\}$. 
We may regard $F$ as a morphism $F: X \rightarrow X$, and will call $F$ the Frobenius map (associated with the rational structure on $X$ ). Some elementary properties of $F$ are the following:

(a) If $F$ and $F^{\prime}$ are $F_{q}, F_{q^{\prime}}$-rational structures on $X$, then there exist $n, n^{\prime} \geqslant 1$ such that $F^{n}=\left(F^{\prime}\right)^{n^{\prime}}$.

(b) Let $g: X \rightarrow X$ be an automorphism of finite order, $F: X \rightarrow X$ a Frobenius map for an $F_{q}$-rational structure, and let $g F=F g$. Then $F g$ is the Frobenius map for some $F_{q}$-rational structure.

(c) Let $Y \subset X$ be a closed subvariety of $X$ (defined by an ideal $I \subset A$ ). Then $F(Y)=Y$ if and only if $F I=I^{q}$. In this case, $F$ defines a homomorphism $F: A / I \rightarrow A^{q} / I^{q}$, and defines an $F_{q}$-rational structure on $Y$.

(d) $F: X \rightarrow X$ is bijective.

(e) The fixed point set $X^{F}=\{x \in X: F(x)=x\}$ is finite (notation: card $X^{F}=\left|X^{F}\right|$ ).

Now let $G$ denote a connected reductive affine algebraic group over $K$, and let $F: G \rightarrow G$ denote a Frobenius map of an $F_{q}$-rational structure on $G$, compatible with the group structure, i.e. $F$ is an endomorphism of affine algebraic groups. Then $G^{F}$ is a finite group. The reductive condition means that $G$ has no nontrivial normal connected unipotent subgroup, and implies that $O_{p}\left(G^{F}\right)=\{1\}$, where $p$ is the characteristic of $K$.

Each finite group $G^{F}$ defined as above has a split $(B, N)$-pair of characteristic $p,\left(B^{F}, N^{F}\right)$, with $B$ an $F$-stable Borel subgroup of $G$, and $N$ the normalizer of an $F$-stable maximal torus $T \subset B$ (the existence of which was proved by Steinberg [77]).

From now on, a finite group of Lie type denotes a group $G^{F}$ arising from a connected reductive group $G$ over $K$ with an $F_{q}$-rational structure defined by a Frobenius map $F$; then $G^{F}$ is a group $G(q)$ belonging to a family of finite groups of Lie type in the sense of \$1.3. It should be noted, however, that more than one family of finite groups of Lie type may arise from a single connected reductive group $G$. For example, let $G=G L_{n}(K)$. For each $p$-power $q$, let $F$ : $G \rightarrow G$ be defined by $F\left(\left(x_{i j}\right)\right)=\left(x_{i j}^{q}\right)$, for each matrix $X=\left(x_{i j}\right)$ belonging to $G$. Then the groups $\left\{G^{F}\right\}$, for different values of $q$, define the family of finite groups of Lie type $\left\{G L_{n}(q)\right\}$. On the other hand, for each $q$, let $F^{\prime}$ denote the Frobenius map defined by $F^{\prime}\left(\left(x_{i j}\right)\right)={ }^{t}\left(x_{i j}^{q}\right)^{-1}$. Then the family of finite groups $\left\{G^{F^{\prime}}\right\}$ for different choices of $q$, are the unitary groups $\left\{U_{n}\left(q^{2}\right)\right\}$, which have a different Weyl group from the groups $G L_{n}(q)$.

Conversely, from the classification it follows that all simple finite groups of Lie type, in the sense of $\S 1.3$, are groups $G^{F}$ constructed as above.

The conjugacy classes in a finite group of Lie type $G^{F}$ are determined as follows. Let $x \in G^{F}$, and let $x=s u$ be the Jordan decomposition of $x$ in $G$, with $s$ semisimple and $u$ unipotent. Then both $s$ and $u$ belong to $G^{F}$. Moreover, the connected centralizer $Z_{G}(s)^{0}$ is a connected reductive $F$-stable subgroup of $G$, and $x \in Z_{G}(s)^{0}$. From this it follows that the conjugacy class of $x$ is to some extent determined by the conjugacy class of the semisimple part $s$ of $x$, and by a unipotent conjugacy class in the connected reductive group $Z_{G}(s)^{0}$.

We require more information about the semisimple elements in $G^{F}$. These are distributed among the $F$-stable maximal tori in $G$. While all maximal tori 
are conjugate in $G$, the $F$-stable maximal tori split up into conjugacy classes relative to the action of the finite group $G^{F}$. The distribution of the $F$-stable tori into $G^{F}$-classes is critical for an understanding of what is to follow, and we begin with an illustration.

First let $G$ be $S L_{2}(\mathbf{C})$, and $F$ the map $\left(x_{i j}\right) \rightarrow\left(\bar{x}_{i j}\right)$, where $\bar{x}$ denotes the complex conjugate of $x$. Then $G^{F}$ is $S L_{2}(\mathbf{R})$. There are exactly 2 classes of $F$-stable maximal tori in $G$ under the action of $G^{F}$, representatives of which are: the split torus

$$
\left\{\left(\begin{array}{cc}
\xi & 0 \\
0 & \xi^{-1}
\end{array}\right), \xi \in \mathbf{C}\right\}
$$

and the compact torus

$$
\left\{\left(\begin{array}{cc}
\cos \theta & \sin \theta \\
-\sin \theta & \cos \theta
\end{array}\right): \theta \in \mathbf{R}\right\} .
$$

The compact torus is obtained from a split torus in $S L_{2}(\mathbf{C})$ as the kernel of the norm map

$$
\left(\begin{array}{cc}
\xi & 0 \\
0 & \xi^{-1}
\end{array}\right) \rightarrow\left(\begin{array}{cc}
N(\xi) & 0 \\
0 & N(\xi)^{-1}
\end{array}\right),
$$

where $N(\xi)=\xi \bar{\xi}=|\xi|^{2}$.

A similar analysis holds for $S L_{2}(K)$, with $F$ the Frobenius map given by $F\left(\left(x_{i j}\right)\right)=\left(x_{i j}^{q}\right)$. Again there are two $S L_{2}(q)$-classes of $F$-stable maximal tori, represented by the split torus (as above) and the precise analogue (defined in terms of the norm map from $F_{q^{2}} \rightarrow F_{q}$ ) of the compact torus. The corresponding subgroups of $S L_{2}(q)$ have orders $q-1$ and $q+1$, respectively.

For the classification of $F$-stable maximal tori for a general $F_{q}$-rational structure, we require Lang's theorem, which asserts that the map $x \rightarrow$ $x^{-1} F(x)$, from a connected group $G$ with Frobenius map $F$, into itself, is surjective. The main result is as follows.

(4.1.1) Theorem. Let $G$ be connected, reductive, with Frobenius map $F$.

(i) $G$ contains a maximal torus fixed by $F$.

(ii) If $T$ is such a maximal torus, and $W=N / T$ is the Weyl group, then the classes of maximal $F$-stable tori under conjugation by $G^{F}$, are in bijective correspondence with the elements of $H^{1}(F, W)$, where $H^{1}(F, W)$ is the set of equivalence classes in $N / T$ derived from the relation $n \sim n^{\prime}$ in $N$ if $n=$ $n^{\prime \prime} n^{\prime} F\left(n^{\prime \prime}\right)^{-1}$, for some $n^{\prime \prime} \in N$.

(iii) If $F$ commutes with the action of $W$ on $T$, then the $G^{F}$-classes of $F$-stable tori are in bijective correspondence with the conjugacy classes in $W$.

(iv) Let $w \in W$. An F-stable maximal torus corresponding to the class of $w$ in $H^{1}(F, W)$ is $\mathrm{gTg}^{-1}$, where $g$ is an element (whose existence follows from Lang's theorem) satisfying $g^{-1} F(g)=n_{w}$, where $n_{w} \in N$ is a representative of the coset $w \in N / T$.

4.2 Cuspidal characters of $G L_{n}(q)$ via the Brauer lift. The Steinberg representation. This section provides an introduction to the use of homological methods in the representation theory of finite groups of Lie type. We first give an account of a new version of Green's construction of irreducible 
cuspidal characters of $G L_{n}(q)$ using the Brauer lift of the identity representation. The main result has a topological interpretation, as one might be led to expect from the earlier construction by Solomon and Tits of the Steinberg representation on the rational homology of the simplicial building of a finite group of Lie type. We give a sketch of the method as it applies to $\mathrm{St}_{G}$, and refer to Lusztig [49] (see also Springer [67]) for the applications to $G L_{n}(q)$.

Let $G$ be a finite group, and let $S: G \rightarrow G L(M)$ be a representation of $G$ by invertible linear transformations on a finite-dimensional vector space over a finite field, and let $E$ be a finite extension of the field containing the eigenvalues of all elements $\{S(g), g \in G\}$. Let $\theta: E^{*} \rightarrow \mathbf{C}^{*}$ be an injective homomorphism of the multiplicative group of $E$ into the multiplicative group of the complex numbers.

Definition. Let $G, S: G \rightarrow G L(M), \theta$ be as above. Define a complex valued function $\beta_{\theta, S}$ on $G$ by the formula

$$
\beta_{\theta, S}(g)=\theta\left(\xi_{1}\right)+\cdots+\theta\left(\xi_{n}\right), \quad g \in G,
$$

where $\left\{\xi_{1}, \ldots, \xi_{n}\right\}$ are the eigenvalues of $S(g)$ and $n=\operatorname{dim} M$. The function $\beta_{\theta, S}$ is called the Brauer lift of the representation $S$.

(4.2.1) TheOREM (GReEN). The Brauer lift $\beta_{\theta, S}$ defined above is a virtual character of $G$.

The proof uses Brauer's Theorem 2.1.3, and involves checking the restrictions of $\beta_{\theta, S}$ to the elementary subgroups of $G$.

Now let $G=G L_{n}(K)$, and $F: G \rightarrow G$ the Frobenius map such that $G^{F} \cong$ $G L_{n}(q)$. In this case the Brauer lift of the identity representation $I: x \rightarrow I(x)$ $=x$ of $G^{F}$ satisfies the condition $\beta_{\theta, I}(x)=\beta_{\theta, I}(s)$ if $s$ is the semisimple part of $x \in G^{F}$. Concerning such functions on $G^{F}$, Kawanaka proved the following useful result.

(4.2.2) THEOREM. Let $G^{F}$ be a finite group of Lie type (as in \$4.1), and let $\psi$ : $G^{F} \rightarrow \mathbf{C}$ be a function such that for all $x, \psi(x)=\psi(s)$, where $s$ is the semisimple part of $x \in G^{F}$. Then

$$
\sum_{x \in G} \psi(x)=\sum_{T} \sum_{t \in T^{F}} \psi(t)
$$

where $\{T\}$ is a set of representatives of the $G^{F}$-conjugacy classes of $F$-stable maximal tori in $G$.

(4.2.3) Corollary. Let $G=G L_{n}(q)$, and let $\beta_{\theta, I}$ be the Brauer lift of the identity representation. Then

$$
\left(\beta_{\theta, I}, \beta_{\theta, I}\right)_{G}= \begin{cases}n & \text { if } q>2 \\ n+1 & \text { if } q=2 .\end{cases}
$$

The problem now is to find the irreducible components of $\beta_{\theta, I}$, hoping to find a cuspidal one. The method is as follows. A flag in the underlying vector space over $F_{q}$ is a chain of subspaces

$$
\{0\} \subset M_{1} \subset M_{2} \subset \cdots \subset M_{d}=M .
$$

A flag is complete if $\operatorname{dim}\left(M_{i+1} / M_{i}\right)=1$ for all $i$. The Borel subgroups in 
$G L_{n}(q)$ are the stabilizers of complete flags. For a fixed complete flag as above, with $d=n=\operatorname{dim} M$, let $P_{i}=\operatorname{Stab}_{G}\left(M_{i}\right), 1 \leqslant i \leqslant n-1$, where $G=$ $G L(M)$. Each $P_{i}$ is a maximal parabolic subgroup of $G$. Moreover, for each $i$, there is a natural homomorphism $\pi_{i}: P_{i} \rightarrow G L\left(M_{i}\right)$, and we may consider the Brauer lift $\beta_{\theta, I_{M_{i}}}$ of the identity representation of $G L\left(M_{i}\right)$.

(4.2.4) Theorem (Lusztig). Let $G=G L(M)$, and $\beta_{\theta, I}$ the Brauer lift of the identity representation on $M$. Let $0 \subset M_{1} \subset \cdots \subset M_{n}=M$ be a complete flag in $M$, as above.

(i) There exists a unique irreducible cuspidal character $\zeta_{\theta, M}$ which appears with positive multiplicity in $\beta_{\theta, I}$.

(ii) We have

$$
\beta_{\theta, I}=\sum_{i=1}^{n-1}(-1)^{i-1}\left(\zeta_{\theta, M_{i}} \circ \pi_{i}\right)^{G}+(-1)^{n-1} \zeta_{\theta, M}
$$

where $\zeta_{\theta, M_{i}}$ is the unique irreducible cuspidal component (from (i)) of the Brauer lift of the identity representation of $G L\left(M_{i}\right)$, and $\pi_{i}$ is the homomorphism from $P_{i} \rightarrow G L\left(M_{i}\right)$, defined above.

(iii) The characters $\left(\zeta_{\theta, M_{i}} \circ \pi_{i}\right)^{G}$ are irreducible if $i>1$ or if $q>2$. If $q=2$, then $\left(\zeta_{\theta, M_{1}} \circ \pi_{1}\right)^{G}$ is the sum of an irreducible character and the trivial character $1_{G}$.

The formula for the character $\zeta_{\theta, M}$ suggests that the Brauer lift $\beta_{\theta, I}$ might arise from representations of $G$ on the homology of an appropriate complex. Such an interpretation was found by Lusztig [49]. We shall present here the more elementary application of topological methods to the construction of the Steinberg character $\mathrm{St}_{G}$ of a finite group of Lie type, by Solomon and Tits.

Definition. The simplicial building $K_{G}$ of a finite group of Lie type $G^{F}$ is the finite simplicial complex whose vertices are the subgroups $\left\{P^{F}\right\}$ corresponding to maximal $F$-stable parabolic subgroups $P$ of $G$. A set of vertices $\left\{P_{0}^{F}, \ldots, P_{r}^{F}\right\}$ defines an $r$-simplex in $K_{G}$ if and only if $P_{0} \cap \cdots \cap P_{r}$ is a parabolic subgroup of $G$.

(4.2.5) THEOREM. Let the rank $n$ of the $(B, N)$-pair in $G^{F}$ be at least two. Then the underlying topological space of $K_{G}$ has the homotopy type of a bouquet of spheres, each of dimension $n-1$. The rational homology groups of $K_{G}$ satisfy: $H_{i}\left(K_{G}\right)=0,0<i<n-1, \operatorname{dim}_{Q} H_{0}\left(K_{G}\right)=1$, and $\operatorname{dim}_{Q} H_{n-1}\left(K_{G}\right)=$ $\left|G^{F}\right|_{p}$, the p-part of the order of the finite group $G^{F}$, where $p$ is the characteristic.

The group $G^{F}$ acts on the simplicial building $K_{G}$ via conjugation as a group of simplicial automorphisms, and hence acts on the rational homology groups.

(4.2.6) THEOREM. The representation of $G^{F}$ on the top homology group $H_{n-1}\left(K_{G}\right)($ for $n \geqslant 2)$ is the Steinberg representation of $G^{F}($ see $\$ 3.2)$. We have

$$
\mathrm{St}_{G}=\sum_{J}(-1)^{|J|} 1_{P_{J}^{F}}^{G^{F}}
$$


where $\left\{P_{J}\right\}$ is a complete set of standard parabolic subgroups of $G$, parametrized by the parabolic subgroups $\left\{W_{J}\right\}$ of the Weyl group $W$.

Springer was the first to recognize that the values of $\mathrm{St}_{G}$ can also be found by topological methods. Here is a sketch of a new approach to this problem.

(4.2.7) LemMA. Let $K$ be a finite simplicial complex, and $G$ a finite group of simplicial automorphisms of $K$. Let $\Lambda$ be the Lefschetz character of $G$ on the rational homology of $K$, defined by $\Lambda(x)=\Sigma(-1)^{i} \operatorname{Tr}\left(x, H_{i}(K)\right)$, for $x \in G$. For each $x \in G, \Lambda(x)$ is the Euler characteristic $\chi\left(|K|^{x}\right)$ of the fixed point set $|K|^{x}$ of $x$ acting on the underlying topological space $|K|$ of $K$.

(4.2.8) THEOREM. Let $G^{F}$ be a finite group of Lie type, for a connected reductive group $G$ with an $F_{q}$-rational structure and Frobenius map $F$, and let $\mathrm{St}_{G}$ be the character of the Steinberg representation of $G^{F}$. The values of $\mathrm{St}_{G}$ are given as follows:

(i) $\mathrm{St}_{G}(x)=0$ for all nonsemisimple elements of $x \in G^{F}$.

(ii) $\mathrm{St}_{G}(x)=\mathrm{St}_{L}(x)$, if $x \in G^{F}$ is such that $Z_{G}(x) \leqslant L$, for an $F$-stable Levi factor $L$ of an $F$-stable parabolic subgroup $P$ of $G$.

(iii) For all semisimple elements $s \in G^{F}$,

$$
\mathrm{St}_{G}(s)=\varepsilon_{G} \varepsilon_{Z_{G}(s)} \mathrm{St}_{Z_{G}(s)}{ }^{0}(1),
$$

where for a connected reductive $F$-stable subgroup $H$ of $G, \varepsilon_{H}=(-1)^{\sigma(H)}$, and $\sigma(H)$ is the dimension of a maximal $F_{q}$-split torus in $H$.

We shall give an indication of the method of proof, to show how Lemma 4.2.7 is used. By (4.2.5) and (4.2.6), deg $\mathrm{St}_{G}=\left|G^{F}\right|_{p}$, so that $\mathrm{St}_{G}$ belongs to a $p$-block of defect zero, and vanishes on nonsemisimple elements by a theorem of Brauer and Nesbitt. Statement (ii) follows from a reduction theorem which applies to all characters in $1_{B^{F}}^{G^{F}}$ (see (4.4.5)). For semisimple elements $s$ satisfying the hypothesis of (ii), statement (iii) follows by induction on the rank of a $(B, N)$-pair in $G^{F}$. Now let $s \in G^{F}$ be an element whose connected centralizer $Z_{G}(s)^{0}$ is contained in no proper $F$-stable parabolic subgroup of $G$. In this case, the homology groups of the fixed point set $\left(K_{G}\right)^{s}$ are isomorphic to the corresponding homology groups of the simplicial building $K_{Z_{G}(s)^{0}}$ of the connected reductive $F$-stable subgroup $Z_{G}(s)^{0}$. Using (4.2.5), (4.2.6) and (4.2.7) we have

$$
\begin{aligned}
\Lambda(s) & =1+(-1)^{n-1} \operatorname{St}_{G}(s) \\
& =\chi\left(K_{Z_{G}(s)^{0}}\right)=1+(-1)^{m-1} \operatorname{St}_{Z_{G}(s)^{0}}(1) .
\end{aligned}
$$

Therefore

$$
\mathrm{St}_{G}(s)=(-1)^{n-m} \mathrm{St}_{Z_{G}(s)^{o}}(1),
$$

where $n$ and $m$ are the $(B, N)$-ranks of $G^{F}$ and $Z_{G}(s)^{0 F}$ respectively. Because $Z_{G}(s)^{0}$ is contained in no proper $F$-stable parabolic subgroup of $G$, we have $n-m=\sigma(G)-\sigma\left(Z_{G}(s)^{0}\right)$, and the result follows.

The character values of the cuspidal characters $\zeta_{\theta, M}$ of $G L_{n}(q)$ were computed by Lusztig [49].

4.3 Representations on l-adic cohomology. The solution of the MacDonald 
conjectures. Let $X$ be an affine algebraic variety with an $F_{q}$-rational structure, and Frobenius map $F: X \rightarrow X$. For every $n, F^{n}: X \rightarrow X$ is an $F_{q^{n} \text {-rational }}$ structure, and from $\S 4.1,\left|X^{F^{n}}\right|<\infty$ for $n=1,2, \ldots$. For example, if $X$ is defined by polynomial equations with coefficients in $F_{q}$, and $F$ is the Frobenius map which raises coordinates of points in $K^{n}$ to $q$ th powers, then $\left|X^{F^{n}}\right|$ is simply the number of solutions of these equations in the finite field $F_{q^{n}}$. In order to understand the numbers $\left|X^{F^{n}}\right|$, in general, we introduce the generating function $\Sigma_{1}^{\infty}\left|X^{F^{n}}\right| t^{n-1}$, viewed as a power series in the complex variable $t$. In his fundamental paper [82], Weil conjectured (among other things) that the above series can be expressed in the form

$$
\sum_{1}^{\infty}\left|X^{F^{n}}\right| t^{n-1}=\frac{d}{d t} \log Z(t)
$$

where $Z(t)$ is a certain rational function in $t$, called the zeta function of the variety $X$.

Weil also conjectured that the properties of $Z(t)$ could be established by cohomological methods. This has been accomplished, and we shall require some insight towards these methods in order to proceed with the character theory.

Let $g: X \rightarrow X$ be an automorphism of finite order commuting with $F$. From $\S 4.1, g F$ is also a rational structure on $X$. It is possible to introduce cohomology groups $H_{c}^{i}\left(X, \bar{Q}_{l}\right)$, called the l-adic cohomology groups on $X$ with compact supports, with coefficients in the algebraic closure $\bar{Q}_{l}$ of the field of $l$-adic numbers for some prime $l \neq p$, such that the number of fixed points $\left|X^{F^{n}} g\right|$ is given by the Lefschetz number of $F^{n} g$ on the $l$-adic cohomology. More precisely, we have

$$
\left|X^{F^{n} g}\right|=\sum_{i=0}^{\infty}(-1)^{i} \operatorname{Tr}\left(F^{n} g, H_{c}^{i}\left(X, \bar{Q}_{l}\right)\right) .
$$

(To interpret the sum, we require the facts that the groups $H_{c}^{i}\left(X, \overline{Q_{l}}\right)$ are finite dimensional vector spaces over $Q_{l}$, and vanish for large values of $i$.)

Now let $H_{c}^{i}\left(X, \bar{Q}_{l}\right)_{\lambda}$ denote the generalized eigenspace in $H_{c}^{i}\left(X, \bar{Q}_{l}\right)$ for the eigenvalue $\lambda$ of $F$. Because $F$ commutes with $g$, it follows that

$$
\begin{aligned}
\left|X^{F^{n}}\right| & =\sum_{i}(-1)^{i} \sum_{\lambda} \lambda^{n} \operatorname{Tr}\left(g, H_{c}^{i}\left(X, \overline{Q_{l}}\right)_{\lambda}\right) \\
& =\sum_{\lambda} \lambda^{n}\left(\sum_{i}(-1)^{i} \operatorname{Tr}\left(g, H_{c}^{i}\left(X, \bar{Q}_{l}\right)_{\lambda}\right)\right) \\
& =\sum a_{\lambda} \lambda^{n}
\end{aligned}
$$

where

$$
a_{\lambda}=\sum(-1)^{i} \operatorname{Tr}\left(g, H_{c}^{i}\left(X, \bar{Q}_{l}\right)\right)_{\lambda} .
$$

Then the generating function for the numbers $\left|X^{F^{n} g}\right|$ is given, after some manipulation, by

$$
-\sum_{1}^{\infty}\left|X^{F^{n} g}\right| t^{n}=-\sum_{\lambda, n} a_{\lambda} x^{n} t^{n}=\sum_{\lambda} a_{\lambda} \frac{1}{1-(\lambda t)^{-1}} .
$$


This shows how the rationality of the zeta function is proved in this case, and also calls attention to the following objects.

Definition. Let $X, F, G, H_{c}^{i}\left(X, \overline{Q_{l}}\right)$, etc. be as above. The Lefschetz number $\mathcal{L}(g, X)$ is defined by

$$
\mathfrak{L}(g, X)=\sum(-1)^{i} \operatorname{Tr}\left(g, H_{c}^{i}\left(X, \bar{Q}_{l}\right)\right) .
$$

From the above discussion, we see that $\mathcal{L}(g, X)=\Sigma_{\lambda} a_{\lambda}$, and therefore obtain the following result, which expresses the Lefschetz number in terms of the sequence $\left|X^{F^{n} g}\right|, n=1,2, \ldots$

(4.3.1) Proposition.

$$
\mathcal{L}(g, X)=-\left(\sum_{n=1}^{\infty}\left|X^{F^{n} g}\right| t^{n}\right)_{t=\infty} .
$$

From (4.3.1), several basic properties of $\mathcal{L}(g, X)$ can be derived. In fact, (4.3.1) suggests that the Lefschetz numbers can be investigated independently of $l$-adic cohomology. At the present time, however, some essential properties of the Lefschetz numbers require a cohomological proof.

Now let $G$ be a connected reductive group, with Frobenius endomorphism $F$ for an $F_{q}$-rational structure on $G$. A fundamentally new method in the representation theory of the finite groups $G^{F}$, introduced first by Drinfeld in the case of $S L_{2}(q)$, and by Deligne and Lusztig in general, leads to the construction of families of virtual characters of $G^{F}$, defined in terms of the Lefschetz numbers associated with the action of $G^{F}$ on certain affine algebraic varieties. The results obtained from this construction give a complete solution to a remarkable set of conjectures made by MacDonald in 1968, on the basis of character tables known at the time, and his understanding of parallel theories for semisimple groups over real and $p$-adic fields. What follows is a summary of a new version by Lusztig [56] of the Deligne-Lusztig construction, and the solution of the MacDonald conjectures.

Let $T \subset G$ be an $F$-stable maximal torus, and let $\theta: T^{F} \rightarrow \mathrm{C}$ be a fixed linear character of the finite group $T^{F}$. For each such pair $(T, \theta)$, we shall construct a virtual character $R_{T}(\theta)$ of $G$ (with values in $\mathbf{C}$ ) as follows. Let $B$ be a Borel subgroup of $G$ containing $T$ (not necessarily $F$-stable) and let $U$ be the unipotent radical of $B$.

Lang's theorem asserts that the morphism $L: G \rightarrow G$, given by $L(g)=$ $g^{-1} F(g)$, is surjective. The set

$$
\tilde{X}=\left\{g \in G: g^{-1} F(g) \in U\right\}=L^{-1}(U)
$$

is a Zariski closed subset of $G$, and hence is an affine algebraic variety. Moreover, the finite group $G^{F} \times T^{F}$ acts on $\tilde{X}$ via $(g, t) g^{\prime}=g g^{\prime} t^{-1}$, for $(g, t) \in G^{F} \times T^{F}$ and $g^{\prime} \in \tilde{X}$. A basic property of the Lefschetz numbers is that the map

$$
(g, t) \rightarrow \mathfrak{L}((g, t), \tilde{X})
$$

is a virtual character of $G^{F} \times T^{F}$ with values in $Z$.

Definition. The Deligne-Lusztig virtual character associated with the pair $(T, \theta)$ is the map 


$$
R_{T, U}(\theta): g \rightarrow \frac{1}{\left|T^{F}\right|} \sum_{t \in T^{F}} \mathcal{L}((g, t), \tilde{X}) \theta(t),
$$

defined for $g \in G^{F}$.

It is not difficult to show that $R_{T, U}(\theta)$ is a virtual character of $G^{F}$, and it will follow from the orthogonality relations that $R_{T, U}(\theta)$ is independent of $U$.

The existence of families of virtual characters of $G^{F}$ parametrized by pairs $\{T, \theta\}$, with $T$ a maximal $F$-stable torus, and $\theta$ a character of $T^{F}$, was first proved by Green for the groups $G L_{n}(q)$, and was then established by Srinivasan for the groups $\mathrm{Sp}_{4}(q)$, and by Chang and Ree for the groups $G_{2}(q)$. Their existence and properties in the general case were the contents of the MacDonald conjectures.

For two $F$-stable maximal tori $T, T^{\prime}$, let

$$
N\left(T, T^{\prime}\right)=\left\{n \in G: n^{-1} T n=T^{\prime}\right\} .
$$

Then $T$ acts on $N\left(T, T^{\prime}\right)$ the left and $T^{\prime}$ on the right, so that the orbit spaces $T \backslash N\left(T, T^{\prime}\right)$ and $N\left(T, T^{\prime}\right) / T^{\prime}$ coincide, and will be denoted by $W\left(T, T^{\prime}\right)$. In particular $N(T, T)=N_{G}(T)$, and $W(T, T)$ is the Weyl group $N_{G}(T) / T$ associated with $T$.

In case $T$ and $T^{\prime}$ are $F$-stable, $N\left(T, T^{\prime}\right)$ is $F$-stable, and using Lang's theorem, it can be proved that

$$
T^{F} \backslash N\left(T, T^{\prime}\right)^{F}=N\left(T, T^{\prime}\right)^{F} / T^{\prime F}=W\left(T, T^{\prime}\right)^{F} .
$$

We can now state the first fundamental property of the Deligne-Lusztig characters.

(4.3.2) Theorem (OrThogonality formula). Let $T, T^{\prime}$ be F-stable maximal tori in $G$, and let $\theta, \theta^{\prime}$ be linear characters on the finite groups $T^{F}$ and $T^{\prime F}$. Then

$$
\left(R_{T, U}(\theta), R_{T^{\prime}, U^{\prime}}\left(\theta^{\prime}\right)\right)_{G^{F}}=\left|\left\{w \in W\left(T, T^{\prime}\right):{ }^{w} \theta=\theta^{\prime}\right\}\right| .
$$

Note in particular that the above scalar product is zero if $T$ and $T^{\prime}$ are not $G^{F}$-conjugate.

(4.3.3) Corollary. $R_{T, U}(\theta)$ is independent of $U$ (and hence will be written as $R_{T}(\theta)$, or $R_{T}^{G}(\theta)$ if the dependence on $G$ as well as $T$ needs to be shown).

(4.3.4) CoRollary. $R_{T}(\theta)$ is an irreducible character of $G^{F}$ if and only if ${ }^{w} \theta=\theta$, for $w \in W(T, T)^{F}$, holds exactly when $w=1$.

A character $\theta$ satisfying the condition of (4.3.4) is said to be in general position.

Let us consider the special case where $T$ is a maximal $F_{q}$-split torus in $G$, so that $T$ is a Levi factor of an $F$-stable Borel subgroup $B$ of $G$. Then we have, for $\theta: T^{F} \rightarrow \mathbf{C}$,

$$
R_{T}(\theta)=\tilde{\theta}^{G},
$$

so that the irreducible components of $R_{T}^{G}(\theta)$ are precisely the principal series characters considered in \$3.

From a result on the behavior of the Lefschetz numbers $\mathcal{L}(g, X)$ relative to the Jordan decomposition of $g$, the following result can be proved. 
(4.3.5) THEOREM. Let $T \subset G$ be a maximal F-stable torus, and $\theta: T^{F} \rightarrow \mathbf{C} a$ character of $T^{F}$. Let $u$ be a unipotent element in $G^{F}$. Then $\operatorname{Tr}\left(u, R_{T}^{G}(\theta)\right)$ is an integer, independent of $\boldsymbol{\theta}$.

Thus the virtual characters $R_{T}^{G}(\theta)$, for a fixed unipotent element $u \in G^{F}$, are constant for all characters $\theta: T^{F} \rightarrow \mathrm{C}$, and define what are called the Green's functions

$$
Q_{T}: u \rightarrow \operatorname{Tr}\left(u, R_{T}(\theta)\right)
$$

corresponding to the various $F$-stable tori $\{T\}$.

The Green's functions satisfy orthogonality relations and other properties which were predicted by Kilmoyer and Springer. Using the Lie algebra of $G$, Springer defined certain functions on the unipotent elements of $G^{F}$, and conjectured that they could be extended to virtual characters of $G^{F}$. This conjecture was proved by Kazdhan, for sufficiently large $q$, with Springer's functions becoming Green's functions. Kazdhan thereby succeeded in giving an alternative construction of the virtual characters $\left\{R_{T}(\theta)\right\}$. Using the Green's functions we have:

(4.3.6) Theorem (ChARACTER FORMUlA). Let $T$ be an $F$-stable maximal torus, and $\theta$ a character of $T^{F}$. Let $x=s u$ be an element of $G^{F}$, expressed in its Jordan decomposition. Then

$$
\operatorname{Tr}\left(x, R_{T}(\theta)\right)=\left(\left|Z_{G}(s)^{0 F}\right|\right)^{-1} \sum_{g \in G^{F}} Q_{T}^{Z_{G}\left(g s g^{-1}\right)^{0}}\left(g u g^{-1}\right) \theta\left(g s g^{-1}\right),
$$

where the sum is taken over all $g \in G^{F}$ such that gsg ${ }^{-1} \in T$, and $Q_{T}^{Z_{G}\left(\mathrm{gsg}^{-1}\right)^{0}}$ denotes the Green's function corresponding to $T$ for the connected reductive group $Z_{G}\left(g s g^{-1}\right)^{0}$.

It is not immediate from the above formula even how to compute the degrees of the virtual characters $\left\{R_{T}(\theta)\right\}$. For this calculation, it is necessary to use the properties of the Steinberg character $\mathrm{St}_{G}$ (see 84.2). In particular, we recall from $\$ 4.2$ the definition of the numbers $\varepsilon_{H}$, for connected reductive $F$-stable subgroups $H$ of $G$.

(4.3.7) THEOREM. The degrees of the virtual characters $R_{T}(\theta)$ are given by

$$
\operatorname{deg} R_{T}(\theta)=\operatorname{Tr}\left(1, R_{T}(\theta)\right)=Q_{T}(1)=\varepsilon_{G^{\prime}} \varepsilon_{T}\left|G^{F}\right| p^{\prime}\left|T^{F}\right|^{-1},
$$

where $\left|G^{F}\right|_{p^{\prime}}$ is the part of the order of $G^{F}$ prime to the characteristic $p$.

In particular, for a maximal $F_{q}$-split torus this formula becomes (as it should from our identification of $R_{T}(\theta)$ with $\tilde{\theta}^{G^{F}}$ )

$$
\operatorname{deg} R_{T}(\theta)=\left|G^{F}\right| p^{\prime}\left|T^{F}\right|^{-1}=\left|G^{F}: B^{F}\right|,
$$

where $B$ is an $F$-stable Borel subgroup of $G$ containing $T$.

The character values on arbitrary elements are still not well understood because the Green's functions $Q_{T}$ are not known in any explicit sense except in certain cases. On semisimple elements, however, the values of the characters $R_{T}(\theta)$ can be given explicitly in terms of the values of Steinberg character (\$4.2) as follows. 
(4.3.8) Corollary. Let $T, \theta$ be as above, and let $s$ be a semisimple element of $G^{F}$. Then

$$
\varepsilon_{T} \varepsilon_{G} \operatorname{Tr}\left(s, R_{T}(\theta)\right) \cdot \operatorname{Tr}\left(s, \mathrm{St}_{G}\right)=\operatorname{Tr}\left(s, \theta^{G^{F}}\right)
$$

where $\theta^{G^{F}}$ is the induced character from $T^{F}$ to $G^{F}$ of $\theta$.

Because the Steinberg character vanishes off the semisimple elements, the above corollary can also be stated in the form

$$
\varepsilon_{T} \varepsilon_{G} R_{T}(\theta) \cdot \mathrm{St}_{G}=\theta^{G} .
$$

(We could have suspected that the induced characters $\theta^{G}$ are important, from the beginning. They vanish however on all the unipotent elements and give no hint of how to recover the lost information connected with character values on unipotent elements.)

Remembering that the point of the whole exercise is to find the cuspidal characters, we are led, following Deligne and Lusztig, to the following results.

Let $P$ be an $F$-stable parabolic subgroup of $G$, with an $F$-stable Levi factor $L$, and unipotent radical $V$. Then

$$
1_{V^{F}}^{G^{F}}=\frac{1}{\left|L^{F}\right|_{p}} \sum_{T \subset L} \sum_{\theta} \varepsilon_{T} \varepsilon_{L} R_{T}(\theta)
$$

where the sum is taken over all $F$-stable maximal tori $T \subset L$, and all characters $\theta: T^{F} \rightarrow \mathrm{C}$. From this result it can be proved without too much difficulty that we have:

(4.3.8) Proposition. An irreducible $G^{F}$-character $\zeta$ is cuspidal if and only if $\left(\zeta, R_{T}(\theta)\right)=0$ for all $F$-stable maximal tori $T$ contained in some proper $F$-stable parabolic subgroup of $G$ and all characters $\theta: T^{F} \rightarrow \mathbf{C}$.

From the previous result, we obtain the following contribution to the main problem of finding the cuspidal characters of $G^{F}$.

(4.3.9) TheOREM. Let $T$ be an F-stable maximal torus, and $\theta: T^{F} \rightarrow \mathrm{C} a$ character of $T^{F}$ in general position. Then $\varepsilon_{T} \varepsilon_{G} R_{T}(\theta)$ is an irreducible character of $G^{F}$, and is cuspidal if and only if $T$ is not contained in any proper $F$-stable parabolic subgroup of $G$.

4.4 Unipotent characters. The virtual characters $R_{T}(1)$ are of special interest, because they are given in terms of the Lefschetz numbers without intervention of characters of $T^{F}$,

$$
R_{T}(1)=\left|T^{F}\right|^{-1} \sum_{t \in T^{F}} \mathcal{L}((g, t), X),
$$

and hence are related directly to the geometry of the underlying varieties $\tilde{X}$. Their irreducible components are called unipotent characters of $G^{F}$, and their role was described by Lusztig as follows. "The irreducible representations constructed (in \$4.3) provide only almost all of the representations of our Chevalley groups. These are in some sense similar to the regular semisimple conjugacy classes among all conjugacy classes of a finite Chevalley group. It seems that to understand all irreducible representations without exception, the essential thing is to understand a rather small class of representations (the 
unipotent ones), which play the same role as the unipotent conjugacy classes among all conjugacy classes of a finite Chevalley group."

In particular, for a maximal $F_{q}$-split torus $T$, we have seen that $R_{T}(1)=$ $1_{B}^{G^{F}}$, for an $F$-stable Borel subgroup containing $T$, so that all the irreducible components of this permutation representation, which we described in detail in $\$ 3$, are unipotent representations.

At first, it would appear that the orthogonality formula (4.3.2) separates the unipotent characters from the irreducible components of the virtual characters $R_{T}(\theta)$, for $\theta \neq 1$. However for virtual characters it can happen (and does) that two such characters can have scalar product zero and still have irreducible components in common. Nevertheless, the following result can be proved.

(4.4.1) THEOREM. Let $\rho$ be an irreducible unipotent character of $G^{F}$. Then $\left(\rho, R_{T}(\theta)\right)_{G^{F}}=0$ for all $F$-stable maximal tori $T$ and characters $\theta \neq 1_{T^{F}}$ of $T^{F}$.

Deligne-Lusztig proved in [25] that an irreducible character $\zeta$ of $G^{F}$ is not unipotent if and only if $\zeta$ appears with positive multiplicity in some virtual character $R_{T}(\theta)$ for $\theta \neq 1$.

The following result shows how unipotent characters behave relative to the organization of the characters in terms of the cuspidal ones, described in \$2.2.

(4.4.2) THEOREM. Let $\zeta$ be a noncuspidal irreducible character of $G^{F}$, such that $\left(\zeta, \tilde{\lambda}^{G}\right)>0$ for an irreducible cuspidal character $\lambda$ of a subgroup $L^{F}$, for an $F$-stable Levi factor $L$ of an $F$-stable parabolic subgroup $P$ of $G$. Then $\zeta$ is unipotent if and only if $\lambda$ is unipotent.

Thus the classification of unipotent representations involves, first, the classification of the unipotent cuspidal characters, and then the decomposition of the induced characters $\tilde{\lambda}^{G}$, for unipotent cuspidal characters of subgroups $L^{F}$ arising from $F$-stable Levi factors of proper $F$-stable parabolic subgroups.

The first example of an unipotent cuspidal character was the character $\theta_{10}$, constructed by Srinivasan [71] as part of her determination of the character table of $\mathrm{Sp}_{4}(q)$. Since then, Lusztig has completed the determination of unipotent cuspidal characters (at least for sufficiently large fields), for each family of finite groups of Lie type with indecomposable Weyl groups.

Moreover, he has also proved the following remarkable result, which shows that the theory of generic algebras and generic degrees developed originally for the principal series characters (see §3), can be applied to all irreducible unipotent representations.

(4.4.3) Theorem. Let $G^{F}$ be a finite group of Lie type, let $P$ be a proper $F$-stable parabolic subgroup of $G$, and let $\rho_{1}$ be an irreducible unipotent cuspidal representation of $L^{F}$ for an $F$-stable Levi factor $L$ of $P$. There exists a finite Coxeter group Qf such that the Hecke algebra $H\left(G^{F}, P^{F}, \tilde{\rho}_{1}\right)$ is a specialization of a generic algebra of type W. Moreover, the degrees of the irreducible components of $\tilde{\rho}_{1}^{G}$ (which are unipotent by (4.4.2)), are given in terms of the generic degrees (for suitable specializations) corresponding to the irreducible characters of the Coxeter group W. 
The cases when the situation of (4.4.3) can arise, the types of Coxeter groups थ that can occur, and the specializations of the generic algebras required to determine the Hecke algebra form an intricate and fascinating set of problems which have been completely solved by Lusztig.

Except for the degrees, explicit formulas for all the character values of the irreducible unipotent characters are still not known. Some definitive results in this direction have been found, however, as the following illustrations show.

(4.4.4) THEOREM. Let $\rho$ be an irreducible unipotent character of $G^{F}$, and let $s$ be a regular semisimple element belonging to a maximal $F$-stable torus $T$. Then the value of $\rho$ at $s$ is given by

$$
\rho(s)=\left(\rho, R_{T}(1)\right)_{G^{F}} .
$$

In particular, in case $\rho \in 1_{B}^{G^{F}}$, and $s$ is regular semisimple in an $F$-stable torus $T$ contained in an $F$-stable Borel subgroup $B$, we have, using (4.4.4) and the results of $\S 3$,

$$
\rho(s)=\left(\rho, 1_{B^{F}}^{G^{F}}\right)=\operatorname{deg} \varphi,
$$

where $\varphi$ is the irreducible character of the Weyl group $W$ of $G^{F}$ corresponding to $\rho$ (see Theorem 3.2.6).

For unipotent characters in $1_{B}^{G^{F}}$, there are sharper results. For example:

(4.4.5) THEOREM. Let $\rho$ be an irreducible character in $1_{B}^{G^{F}}$, and let $x \in G^{F}$ be an element such that $Z_{G^{F}}(x) \subseteq L^{F}$ for an $F$-stable Levi factor $L$ of a proper parabolic subgroup $P$ of $G$. Then

$$
\rho(x)=\sum\left(\rho, \tilde{\lambda}^{G}\right) \lambda(x)
$$

where the sum is taken over irreducible characters $\lambda$ of $L^{F}$, which are components of $1_{B_{L}^{F}}^{L^{F}}$, where $B_{L}$ is an F-stable Borel subgroup of $L$. Moreover, the multiplicities $\left(\rho, \tilde{\lambda}^{G}\right)$ are independent of the finite field $F_{q}$, and are given by

$$
\left(\rho, \tilde{\lambda}^{G}\right)=\left(\varphi, \psi^{W}\right)
$$

where $\varphi$ is the irreducible character of the Weyl group corresponding to $\rho$, and $\psi$ is the irreducible character of the parabolic subgroup $W_{L}$ of the Weyl group of $L$ corresponding to $\lambda$.

A deeper result has been proved using cohomological methods by Lusztig.

(4.4.6) THEOREM. Let $\rho$ be an irreducible character in $1_{B}^{G^{F}}$, corresponding to the irreducible character $\varphi$ of the Weyl group $W$ of $G^{F}$. Let $s$ be a regular semisimple element of $G^{F}$ belonging to a maximal F-stable torus $T$ of $G$, corresponding to the conjugacy class $\mathbb{E}$ of $W$ (see (4.1.1)). There exists a virtual character $\xi_{\mathbb{E}}$ of $W$ corresponding to $\mathbb{E}$ such that the value of $\rho$ at $s$ is given by

$$
\rho(s)=\left(\varphi, \xi_{\Subset}\right)_{W} .
$$

In particular, $\rho(s)$ is independent of $q$.

These results provide strong evidence that not only the degrees, but perhaps all the character values of irreducible unipotent characters, can be given generically in some way. 


\section{Guide to the Literature.}

1.

1.1. Borel [6]; Carter [11]; Chevalley [14]; Dickson [26]; Dieudonné [27]; Kostant [47]; Steinberg [76].

1.2 and 1.3. Borel and Tits [8]; Bourbaki [9]; Carter [11]; Fong and Seitz [29]; Richen [59]; Tits [81].

2.

2.2. Curtis [18]; Harish-Chandra [37]; Lusztig [56]; Springer [64], [65], [66].

3.

3.1. Curtis and Fossum [20].

3.2.1. Iwahori [40]; Matsumoto [58].

3.2.2. Bourbaki [9]; Steinberg [76].

3.2.4, 3.2.5, 3.2.6. Benson and Curtis [3]; Curtis, Iwahori, and Kilmoyer [21]; Kilmoyer [44].

Other results related to generic degrees: Benson [2]; Benson, Grove, Surowski [4]; Carter [10]; Curtis [17], [18]; Curtis, Kantor and Seitz [22]; Frame [30], [31]; Green [35]; Hoefsmit [38]; Howlett [39]; Kilmoyer [44]; Kondo [46]; Surowski [78], [79], [80].

Principal series characters. Benson [1]; Kilmoyer [45].

4.

4.1. Borel [5], [6]; Borel and Tits [7]; Chevalley [15]; Lusztig [56]; Steinberg [76], [77].

4.1.1. Springer and Steinberg [70].

4.2. The Brauer Lift, and characters of $G L_{n}(q)$. Gelfand [33]; Green [34]; Kawanaka [41]; Lusztig [49]; Solomon [62], [63]; Springer [64], [67].

The Steinberg character. Curtis [16]; Curtis and Lehrer [23]; Garland [32];

Solomon [61]; Springer [66]; Srinivasan [72]; Steinberg [74], [75], [77].

4.3. The Lefschetz formula and zeta functions. Deligne [24]; Deligne and Lusztig [25]; Grothendieck [36]; Lusztig [56]; Weil [82].

Definitions and properties of $R_{T}(\theta)$. Deligne and Lusztig [25]; Kazhdan [43]; Lusztig [52], [56].

Green's functions. Deligne and Lusztig [25]; Green [34]; Kazhdan [43]; Lusztig [50], [56]; Springer [64], [68], [69]; Srinivasan [73].

4.4. Unipotent representations in general. Curtis [19]; Deligne and Lusztig [25]; Lusztig [51], [53], [54], [56].

Character values. 4.4.4. Deligne and Lusztig [25]; 4.4.5. Curtis [18]; 4.4.6. Lusztig [55].

Centralizers of semisimple elements. Carter [12].

Characters of members of families of Chevalley groups. Chang and Ree [13]; Enomoto [28]; Gelfand [33]; Green [34]; Lusztig [49], [53], [54], [55], [56]; Lusztig and Srinivasan [57]; Springer [64], [67]; Srinivasan [71], [73]; Steinberg [74]. 


\section{REFERENCES}

1. C. T. Benson, On the generic algebra of a unipotent subgroup of a finite Chevalley group, Comm. Algebra (to appear).

2.

3. C. T. Benson and C. W. Curtis, On the degrees and rationality of certain characters of finite Chevalley groups, Trans. Amer. Math. Soc. 165 (1972), 251-273; 202 (1975), 405-406.

4. C. T. Benson, L. C. Grove and D. B. Surowski, Semilinear automorphisms and dimension functions for certain characters of finite Chevalley groups, Math. Z. 144 (1977), 149-159.

5. A. Borel, Linear algebraic groups, Benjamin, New York, 1969.

6. _ Properties and linear representations of Chevalley groups, Part A, Seminar on Algebraic Groups and Related Finite Groups, Lecture Notes in Math., vol. 131, Springer-Verlag, Berlin and New York, 1970.

7. A. Borel and J. Tits, Groupes reductifs, Inst. Hautes Études Sci. Publ. Math. 27 (1965), 55-151.

8. Elements unipotents et sous-groupes paraboliques de groupes reductifs. I, Invent. Math. 12 (1971), 95-104.

9. N. Bourbaki, Groupes et algèbres de Lie, Chaps. 4-6, Actualités Sci. Indust. no. 1337, Hermann, Paris, 1968.

10. R. W. Carter, Conjugacy classes in the Weyl groups, Compositio Math. 25 (1972), 1-59.

11. __ Simple groups of Lie type, Wiley, New York and London, 1972.

12. Centralizers of semisimple elements in finite groups of Lie type, Proc. London Math.

Soc. (3) 37 (1978), 491-507.

13. B. Chang and R. Ree, The characters of $G_{2}(q)$, Istituto Nazionale di Alta Matematica, Symposia Mathematica, vol. 13, 1974, pp. 395-413.

14. C. Chevalley, Sur certains groupes simples, Tôhoku Math. J. 7 (1955), $14-66$.

15. Classification des groupes de Lie algébriques, Paris, 1956/1958.

16. C. W. Curtis, The Steinberg character of a finite group with a $(B, N)$-pair, J. Algebra 4 (1966), 433-441.

17. Nazionale di Alta Matematica, Symposia Mathematica, vol. 13, 1974, pp. 343-355.

18. _ Reduction theorems for characters of finite groups of Lie type, J. Math. Soc. Japan 27 (1975), 666-688.

19. _ A note on the connections between the generalized characters of Deligne and Lusztig and the cuspidal characters of reductive groups over finite fields, J. London Math. Soc. 14 (1976), 405-412.

20. C. W. Curtis and T. Fossum, On centralizer rings and characters of representations of finite groups, Math. Z. 107 (1968), 402-406.

21. C. W. Curtis, N. Iwahori and R. Kilmoyer, Hecke algebras and characters of parabolic type of finite groups with $(B, N)$-pairs, Inst. Hautes Etudes Sci. Publ. Math. 40 (1972), 81-116.

22. C. W. Curtis, W. M. Kantor and G. M. Seitz, The 2-transitive permutation representations of the finite Chevalley groups, Trans. Amer. Math. Soc. 218 (1976), 1-59.

23. C. W. Curtis and G. I. Lehrer, The homology of certain fixed point sets in the Tits complex of a reductive group over a finite field (to appear).

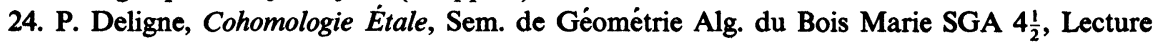
Notes in Math., vol. 569, Springer-Verlag, Berlin and New York, 1977.

25. P. Deligne and G. Lusztig, Representations of reductive groups over finite fields, Ann. of Math. (2) 103 (1976), 103-161.

26. L. E. Dickson, Linear groups, Dover, New York, 1958.

27. J. Dieudonné, La géométrie des groupes classiques, Ergebnisse der Math. und ihrer Grenzgebiete, vol. 5, Springer-Verlag, Berlin and New York, 1955.

28. H. Enomoto, The characters of the finite Chevalley groups $G_{2}(q), q=3^{+}$, Japan. J. Math. (N.S.) 2 (1976), 191-248.

29. P. Fong and G. M. Seitz, Groups with a (B, N)-pair of rank 2. I, II, Invent. Math. 21 (1973). 1-57; 24 (1974), 191-239. 
30. J. S. Frame, The classes and representations of the groups of 27 lines and 28 bitangents, Ann. Mat. Pura Appl. 32 (1951), 83-119.

31. The characters of the Weyl group $E_{8}$, Computational Problems in Abstract Algebra (Proc. Conf. Oxford 1967), ed. J. Leich, Pergamon, Oxford, 1970, pp. 111-130.

32. $\mathrm{H}$. Garland, p-adic curvature and the cohomology of discrete subgroups of p-adic groups, Ann. of Math. (2) 97 (1973), 375-423.

33. S. E. Gelfand, Representation of the full linear group over a finite field, Mat. Sb. 83 (125) (1970), 15-41. (Russian)

34. J. A. Green, The characters of the finite general linear groups, Trans. Amer. Math. Soc. 80 (1955), 402-447.

35. On the Steinberg characters of finite Chevalley groups, Math. Z. 117 (1970), 272-288.

36. A. Grothendieck, Formule de Lefschetz et rationalité des functions $-L$, Sem. Bourbaki 279, Decembre 1964, Benjamin, New York.

37. Harish-Chandra, Eisenstein series over finite fields, Functional Analysis and Related Fields, Springer-Verlag, Berlin and New York, 1970, pp. 76-88.

38. $\mathrm{P}$. Hoefsmit, Representations of Hecke algebras of finite groups with $(B, N)$-pairs of classical type, Ph.D. dissertation, Univ. of British Columbia, Vancouver, 1974.

39. R. B. Howlett, On the degrees of Steinberg characters of Chevalley groups, Math. Z. 135 (1974), 125-135.

40. N. Iwahori, On the structure of the Hecke ring of a Chevalley group over a finite field, J. Fac. Sci. Univ. Tokyo Sect. IA 10 (1964), 215-236.

41. N. Kawanaka, A theorem on finite Chevalley groups, Osaka J. Math. 101 (1973), 1-13.

42. , Unipotent elements and characters of finite Chevalley groups, Osaka J. Math. (to appear).

43. D. Kazhdan, Proof of Springer's hypothesis, Israel J. Math. (to appear).

44. R. Kilmoyer, Some irreducible complex representations of a finite group with a BN-pair, Ph.D. dissertation, M.I.T., 1969.

45. __ Principal series representations of finite Chevalley groups, J. Algebra (to appear).

46. T. Kondo, The characters of the Weyl group of type $F_{4}$, J. Fac. Sci. Univ. Tokyo Sect. I, 11 (1965), 145-153.

47. B. Kostant, Groups over Z, Proc. Sympos. Pure Math., vol. 9, Amer. Math. Soc., Providence, R. I., 1966, pp. 90-98.

48. G. I. Lehrer, Adjoint groups, regular unipotent elements and discrete series characters, Trans. Amer. Math. Soc. 214 (1975), 249-260.

49. G. Lusztig, The discrete series representations of the general linear groups over a finite field, Ann. of Math. Studies, no. 81, Princeton Univ. Press, Princeton, N. J., 1974.

50. __ On the Green polynomials of classical groups, Proc. London Math. Soc. 33 (1976), $443-475$.

51. __ Coxeter orbits and eigenspaces of Frobenius, Invent. Math. 28 (1975), 101-159.

52. $\ldots$ On the finiteness of the number of unipotent classes, Invent. Math. 34 (1976), 201-213.

53. $125-175$.

54. __ Unipotent representations of a finite Chevalley group of type $E_{8}$ (to appear).

55. __ On the reflection representation of a finite Chevalley group (to appear).

56. _ Representations of finite Chevalley groups, CBMS Regional Conf. Ser. in Math., no. 39, Amer. Math. Soc., Providence, R. I., 1978.

57. G. Lusztig and B. Srinivasan, The characters of the finite unitary groups, J. Algebra 49 (1977), 167-171.

58. H. Matsumoto, Générateurs et relations des groupes de Weyl generalise, C. R. Acad. Sci. Paris 258.

59. F. Richen, Modular representations of split $(B, N)$-pairs, Trans. Amer. Math. Soc. 140 (1969), 435-460.

60. G. M. Seitz, Flag transitive subgroups of Chevalley groups, Ann. of Math. (2) 97 (1973), $27-56$. 
61. L. Solomon, The Steinberg character of a finite group with a BN-pair, Theory of Finite Groups (ed. by R. Brauer and C. H. Sah), Benjamin, New York, 1969, pp. 213-221.

62. On the affine group over a finite field, Representation Theory of Finite Groups and Related Topics, Proc. Sympos. Pure Math., vol. 21, Amer. Math. Soc., Providence, R. I., 1971.

63. , The affine group I. Bruhat decomposition, J. Algebra 20 (1972), 512-539.

64. T. A. Springer, Cusp forms for finite groups, and characters of special groups, parts C and D in Seminar on Algebraic Groups and Related Topics (ed. A. Borel) Lecture Notes in Math., vol. 131, Springer-Verlag, Berlin and New York, 1970.

65. , On the characters of certain finite groups, Proc. Summer School, Group Representations, Budapest, 1971.

66. , Caractères de groupes de Chevalley finis, Sem. Bourbaki, no. 429, 1972/73.

67. $\longrightarrow$ Relèvement de Brauer et représentations paraboliques de $G L_{n}\left(F_{q}\right)$, Sem. Bourbaki, no. $441,1973 / 74$.

68. Generalization of Green's polynomials, Proc. Sympos. Pure Math., vol. 21, Amer. Math. Soc., Providence, R. I., 1971, pp. 141-153.

69. Trigonometric sums, Green's functions of finite groups and representations of Weyl groups, Invent. Math. 36 (1976), 173-207.

70. T. A. Springer and R. Steinberg, Conjugacy classes, Part E in Seminar on Algebraic Groups and Related Topics, Lecture Notes in Math., vol. 131, Springer-Verlag, Berlin and New York, 1970.

71. B. Srinivasan, The characters of the finite symplectic group $\operatorname{Sp}(4, q)$, Trans. Amer. Math. Soc. 131 (1968), 488-525.

72. Soc. 12 (1971), 1-14.

73. __ Green polynomials of finite classical groups, Comm. Algebra 5 (1977), 1241-1258.

74. R. Steinberg, A geometric approach to the representations of the full linear group over a Galois field, Trans. Amer. Math. Soc. 71 (1951), 274-282.

75. Prime power representations of finite linear groups. II, Canad. J. Math. 9 (1957), 347-351.

76. , Lectures on Chevalley groups, Yale University, 1967.

77. __ Endomorphisms of linear algebraic groups, Mem. Amer. Math. Soc. no. 80 (1968).

78. D. Surowski, Irreducible characters of BN-pairs of exceptional type, Ph.D. dissertation, Univ. of Arizona, 1975.

79. Representations of a subalgebra of the generic algebra corresponding to the Weyl group of type $F_{4}$, Comm. Algebra 5 (1977), 873-888.

80. , Irreducible characters of BN-pairs of exceptional types, Trans. Amer. Math. Soc. (to appear).

81. J. Tits, Buildings of spherical type and finite BN-pairs, Lecture Notes in Math., vol. 386, Springer-Verlag, Berlin and New York, 1974.

82. A. Weil, Number of solutions of equations in finite fields, Bull. Amer. Math. Soc. 55 (1949), 497-508.

Department of Mathematics, University of Oregon, Eugene, Oregon 97403 